\title{
An efficient multi-scale method for non-linear analysis of composite structures
}

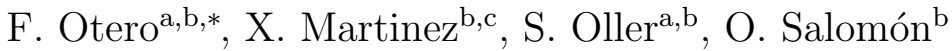 \\ ${ }^{a}$ Departamento de Resistencia de Materiales y Estructuras en la Ingeniería, \\ ETSECCPB, Technical University of Catalonia, Spain \\ ${ }^{b}$ Centre Internacional de Metodes Numerics en Enginyeria (CIMNE), Gran Capitá s/n, \\ 08034 Barcelona, Spain \\ ${ }^{c}$ Departamento de Ciencia e Ingeniería Náutica, FNB, Technical University of \\ Catalonia, Pla de Palau 18, 08003 Barcelona, Spain
}

\begin{abstract}
The use of multi-scale procedures is encouraged by the continuous increase of computational capacity, but it is still a challenge performing a non-linear analysis of a real composite structure without the aid of large computers. This work proposes a strategy to conduct non-linear two-scale analysis in an efficient way. The proposed method considers that in a large structure, in general, material non-linear processes only take place in a localized region (or in a reduced number of finite elements, if a FE method is used). The strategy determines the elements that require a non-linear analysis defining of a non-linear activation function that accounts for the failure of the most critical point in the microstructure. The procedure conserves the dissipated energy through the scales, being mesh independent as the mesh objectivity concept is extended to the microstructure. The validity of the strategy proposed is proved with the analysis of academic examples showing not only the mesh independency but also the reduction of computational cost. Finally, an industrial composite component is solved using a standard computer, showing that the proposed strategy is capable of reducing the computational cost from 32 days and 14 hours (required by a classical multi-scale method) to less than 12 hours.
\end{abstract}

\footnotetext{
${ }^{*}$ Corresponding author at: Centre Internacional de Metodes Numerics en Enginyeria (CIMNE), Gran Capitá s/n, 08034 Barcelona, Spain.

Email addresses: fotero@cimne.upc.edu (F. Otero), x.martinez@upc.edu (X. Martinez), sergio.oller@upc.edu (S. Oller), salomon@cimne.upc.edu (O. Salomón)
} 
Keywords: Composites; Multi-scale homogenization; Non-linear analysis; Finite element method

\section{List of acronyms}

DDM Discrete Damage Mechanics

FE Finite Element

NLAF Non-Linear Activation Function

NLS Non-Linear Strategy

POD Proper Orthogonal Decomposition

RVE Representative Volume Element

SFS Smart First Step

SP Serial Parallel
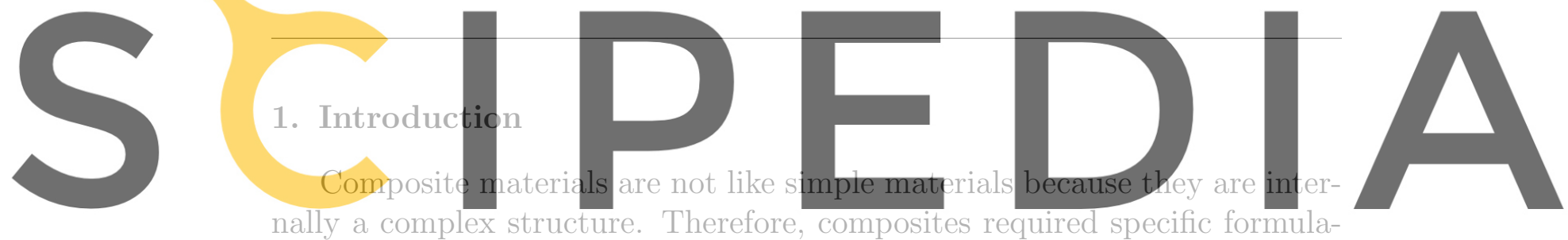

Register for free tions that the ones used in. single materials. For a linear analysis or a study f maximum load it is enough with one orthotropic material characterization

and a law with some complexity to predict the break of the structure (such

as, maximum strain criterion [1], tsai-wu criterion [2], etc). However, numerical methods have allowed the simulation of structures beyond their elastic limit, and then it is possible obtain the post critic behavior of the structure and its tenacity and structural integrity can be estimate.

The complexity of composites has promoted that different formulations appear to predict their behavior, which are more o less suitable according to computational cost available, the accuracy in the results desired or even the expected failure type. In the following, some of the most relevant formulations are described.

One possibility to simulate structures of composite materials which have complex microstructures is to use phenomenological homogenizations. The most common method is the classical mixing theory, which obtains the behavior of the composite from the mechanical performance of the composite components, these are simulated with its own constitutive law [3]. Afterward, 
Oller [4] generalizes the theory to enable the resolution of any composite with reinforced matrix, without the limitation required by the compatibility equation. On the other hand, in the SP [5] continuum approach the mechanic characteristic of the composite are obtained using the properties of each component and taking into account its topological distribution. This serial/parallel (SP) mixing theory assumes a serial-parallel self-adjusting behavior to the topological distribution of fiber embedded in the matrix of the composite material. Recently, one extension of the mixing theory for the study of matrices reinforced with nanotubes has also been developed [6]. All these theories have proved to be able to reproduce not only mechanic properties of composites but to solve non-linear problems also $[7,8,9]$. It is necessary to say that in some particular cases, when the damage is located in one lamina, how in a delamination process, the SP theory may lack accuracy [10].

To simulate laminated composites, one alternative to the previous method-
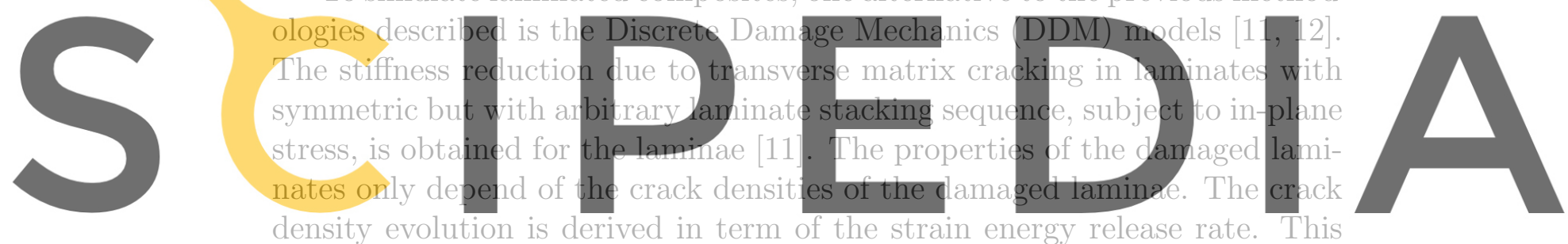

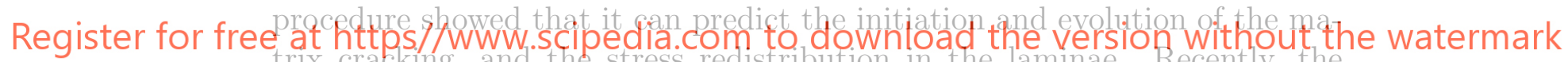

described method was extended to predict the laminate failure throughout

including fiber failure [13]. A Weibull statistical distribution is used to characterize fiber failure and it is incorporated to [11] using a simple fiber damage model. Therefore, the laminate properties now depend of the fiber damage in the laminae too.

On the other hand, Sanchez-Palencia [14] and Suquet [15] laid the foundation of what today is known as first-order homogenization methods. The macroscopic deformation gradient tensor is used to solve the problem at the microstructural scale and then the macroscopic stress tensor is obtained using the averaging equation $[16,17]$. Later, Suquet uses the method of average described to solve and extend the problem of two scales to non-linear range [18]. Over time, thanks to the work to several authors, the method was extended to large deformations with arbitrary non-linear material behavior at the microsctructural scale [19, 20, 21, 22, 23, 24].

Geers and Kouznetsova proposed what is called second-order homogeniza- 
tion $[25,26,27]$, which is an extension of the first-order theory. In this case, the macroscopic deformation gradient tensor and its Lagrangian gradient is used to solve the boundary value problem at the microstructural scale. The second-order approach allows solve problems in the presence of localization phenomena without loss of precision in the solution because the Lagrangian tensor is taken into account. The main drawbacks of this method are its computational cost and complex implementation.

Continuous-discontinuous homogenization method has been developed in the context of masonry [28]. The methodology incorporates a localization band in the macrostructural scale and using the first-order homogenization concepts solve the damaged zone (in the localization band) and the undamaged zone. However, the localization band has a fixed size and need to be located at the beginning. Besides, the approach loses the benefits of the homogenization ideas and it is more similar to a domain decomposition with a refined subscale. Other phenomenological and homogenization models specifically applied to masonry can be found in references

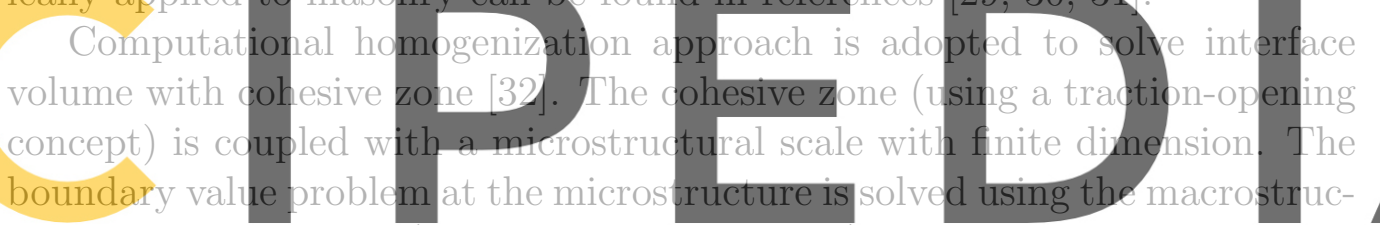

tural scale kinematics (interface opening vector).

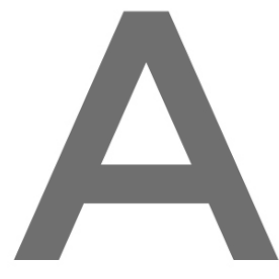

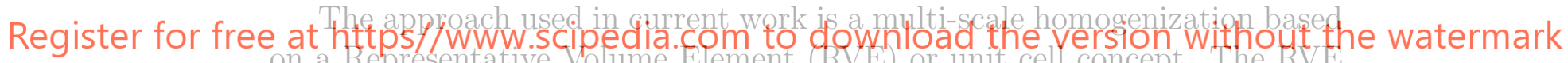

on a Representative Tolume Element (RVE) or unit cell concept. The $\mathrm{RV}$

has a microstructural subregion geometry which is representative of the entire

microstructure. The boundary value problem on the structural scale and in the microstructural scale (RVE) is solved by the Finite Element (FE) method (see AppendixA). With this approach, it is necessary to solve the RVE each time that the macrostructural model requires information about its performance, this is why this kind of solution procedure is known as $\mathrm{FE}^{2}$.

Most of the work on $\mathrm{FE}^{2}$ multi-scale procedures are done on analyzing the numerical performance of RVE [33, 34] or on connecting different scales [35]. In general, in this kind of homogenization methods the elastic properties of the microstructure are obtained solving the microstuctural problem at the beginning of the structure problem. However, the problem with these methods is their computational cost for a non-linear analysis because it is required solving the RVE in every integration point at the macrostructural problem and for every time step to know the non-linear limit and then the behavior of the microstructure in non-linear range. Non-linear performance 
has also the problem that the dissipated energy of both scales is not always related [36].

In order to improve the computational cost of the multi-scale homogenization some strategies use model-order reduction techniques [37, 38, 39]. These methods use the Proper Orthogonal Decomposition (POD) to obtain the reduced set of empirical shape functions. Besides, [39] proved that the common approach of replacing the non-affine term by an interpolant constructed taking only POD modes arrives to ill-posed formulations. An enriched approximation space with the span of the gradient of the empirical shape functions is proposed to avoid this ill-posedness. However, these kind of procedures do not solve the complete structure.

Here it is proposed a new procedure to reduce computational cost of multi-scale simulation. The paper looks also into the problem of localization and energy dissipation across the scales, as the proposed method must be consistent [40]. It is important to note that the procedure developed takes
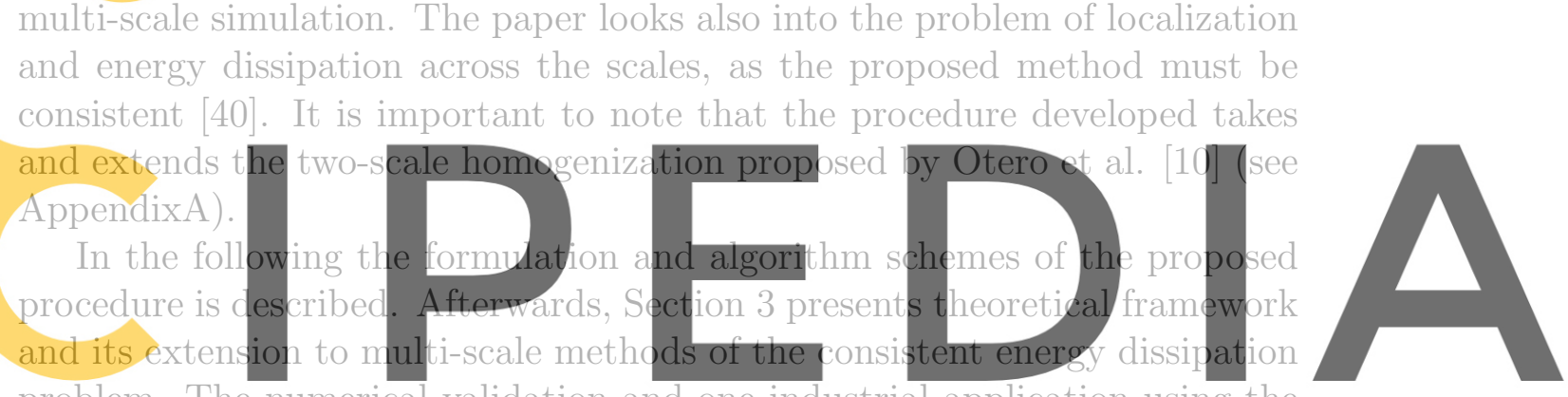

problem. The numerical validation and one industrial application using the

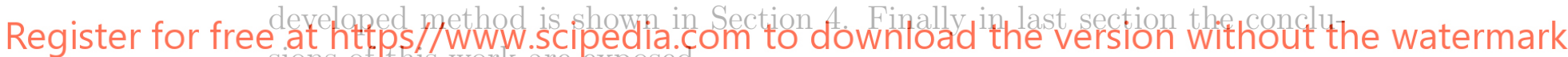

sions of this work are exposed.

\section{New procedure to reduce the computational cost of a multi-scale analysis}

The main advantage of the $\mathrm{FE}^{2}$ method related to a micro model is the reduced computer memory requirements. To solve the same problem, the amount of memory required by the classical FE micro model method is substantially larger than $\mathrm{FE}^{2}$ procedure [10]. This difference is found because the memory used is proportional to the FE mesh size and, while the FE micro model has to solve a problem with a very small discretization, the $\mathrm{FE}^{2}$ procedure only requires memory for the macrostructural problem and the RVE that is being solved. However, if the material reaches non-linear behavior, the computational cost of $\mathrm{FE}^{2}$ method becomes as large as the one required by the micro model case, as the RVE has to be solved for each integration 
point when a real structure is solved. Because of this, a new Non-Linear Strategy (NLS) is proposed in this work.

Continuum mechanics establishes the limit between linear and non-linear performance of materials using comparison criterion that compares a given combination of stresses with a threshold value (Von-Mises, Mohr-Coulomb, etc.). This approach cannot be used in an homogenization double scale solution directly as different strain-stress states may lead to different failure modes of the composite.

A possible solution is to analyze the RVE at every time step. However, this is extremely expensive and very ineffective, because the structural nonlinear behavior often occurs in a small part of its domain.

Here is proposed to develop a comparison function that looks a maximum level of an elastic energy density that can be applied to the RVE before its failure. This is done with the definition of an activation function for each single integration point at the structural scale. It is important to remark
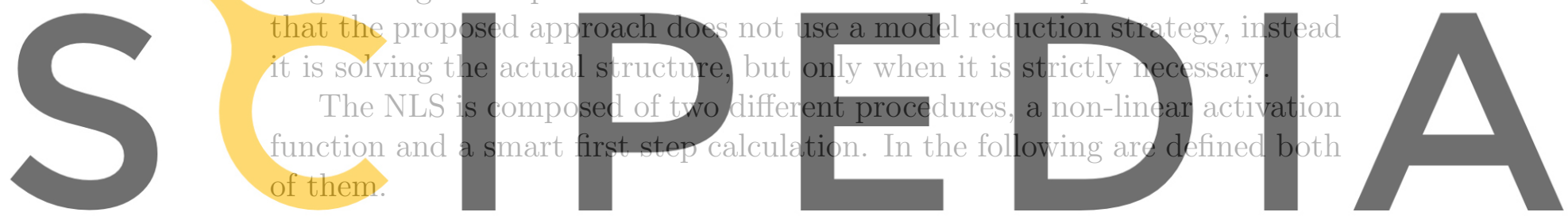

2.1. Non-linear activation function

Register for free athttps/ www.scipedia.com to download the version without the watermark

the fact that any given material begins its non-linear performance when a single particle of the material reaches its stress threshold. The objective of the NLAF is to know whether any material point of the RVE has reached its non-linear limit using homogenized variables.

To do so, present work defines a function $f$ that relates the elastic energy density $\left(\Psi^{e}\right)$ of an integration point of the RVE with the maximum elastic energy $\left(\Psi_{\text {Limit }}^{e}\right)$ that can be applied to this material point, before reaching the non-linear range. Therefore, $f$ is defined as follows:

$$
f=\frac{\Psi^{e}}{\Psi_{\text {Limit }}^{e}} \quad, \quad 0<f<1
$$

In other words, $f$ provides a value of how far is a material point in the microstructure to reach the non-linear state.

In order to know how far is the whole RVE to reach the non-linear performance, it is necessary to use the information obtained for all the integration 
points of the RVE and transform it into a single representative number. This is done with the assumption stated before that the failure of the macrostructure will start when the first integration point of the microstructure fails. Therefore, the $\bar{f}$ parameter of the RVE corresponds to the maximum $f$ value of all integration points of the RVE.

$$
\max \left\{f_{1}, \ldots, f_{n}\right\}=\bar{f}
$$

where the overline at the variables refers to the structure scale or homogenized variables. Finally, the limit elastic energy density at the macrostructure scale is obtained with the following equation:

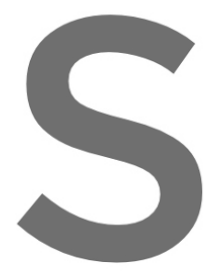

$$
\bar{\Psi}_{\text {Limit }}^{e}=\frac{\bar{\Psi}_{1}^{e}}{\bar{f}}
$$

where $\bar{\Psi}_{1}^{e}$ is the elastic energy density for the strain state used to calculate Register for free at https/Www.scipedia.eom to dow
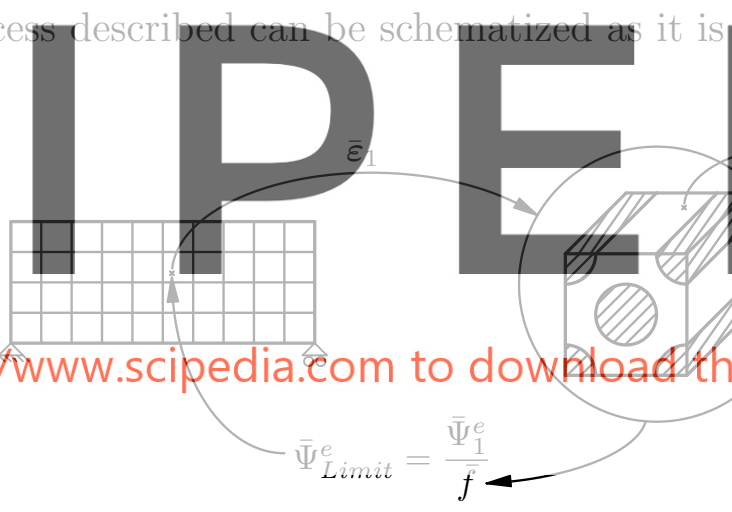

Figure 1: Non-linear activation function scheme.

The NLAF is defined as following:

$$
\bar{\Psi}^{e}-\bar{\Psi}_{\text {Limit }}^{e} \leq 0
$$

where $\bar{\Psi}^{e}$ is the elastic energy density of the macro structural integration point, which is calculated in each load step of the simulation.

The NLAF is valid only for the strain state used to calculate $\bar{\Psi}_{\text {Limit }}^{e}$ (see Eq. 3). If the strain state varies, it may also change the non-linear mode and, therefore, the limit elastic energy density calculated may be no longer valid. Therefore, Eq. (4) is valid while the strain state in the material 
remains proportional to the one used to obtain $\bar{\Psi}_{\text {Limit }}^{e}$. To quantify this proportionality the next equation is proposed:

$$
\frac{\bar{\varepsilon}_{1}: \bar{\varepsilon}_{i}}{\left\|\bar{\varepsilon}_{1}\right\|\left\|\bar{\varepsilon}_{i}\right\|}=1 \Longleftrightarrow \text { Comparable States }
$$

where the subscript $i$ refers to the current i-nth deformation state and $\|$. $\|$ is the norm's mathematical symbol. In case this proportionality is lost, it will be required to calculate again the new limit elastic energy density of the RVE. This is summarized in the following flow diagram (see Fig. 2).

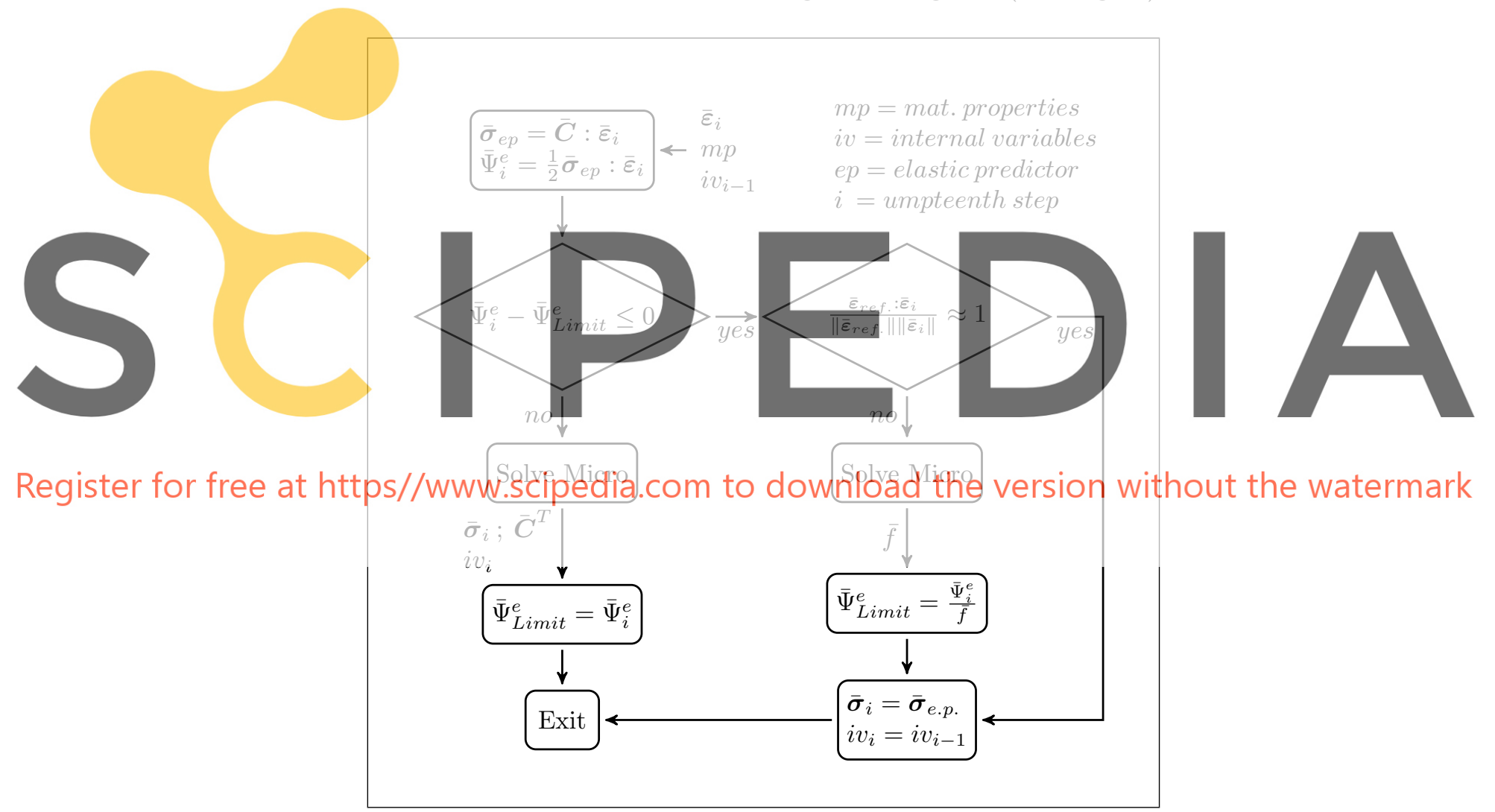

Figure 2: Non-linear strategy algorithm scheme.

It can be easily seen that with the proposed procedure the RVE must be solved for each macro integration point on the first time step, in order to calculate the elastic energy density limit using Eq. (3). Afterwards, it only will be necessary to solve the RVE again if the strain state of the integration point becomes non proportional to the calculated originally or if the NLAF 
is not satisfied, which means that the RVE becomes non-linear. Therefore, if only few elements of the structure reach the non-linear state, only these elements will have to be solved in the non-linear analysis.

\subsection{Smart first step}

As said before, at the beginning of the analysis it is required to solve the RVE for every single integration point of the macrostructure to obtain its $\bar{\Psi}_{\text {Limit }}^{e}$. This calculation process can be extremely expensive in terms of computational cost.

In order to reduce this computational cost, it is proposed a Smart First Step (SFS) strategy. This strategy consist in solving the RVE only if the deformation applied to it is different to all other deformation states considered previously. Therefore before calculating the $\bar{\Psi}_{\text {Limit }}^{e}$ of the RVE, the SFS procedure compares the deformation between the current and the all previous integration points already calculated (Eq. 5). If the SFS finds one comparable strain state, the current RVE takes the values of the RVE already splved.
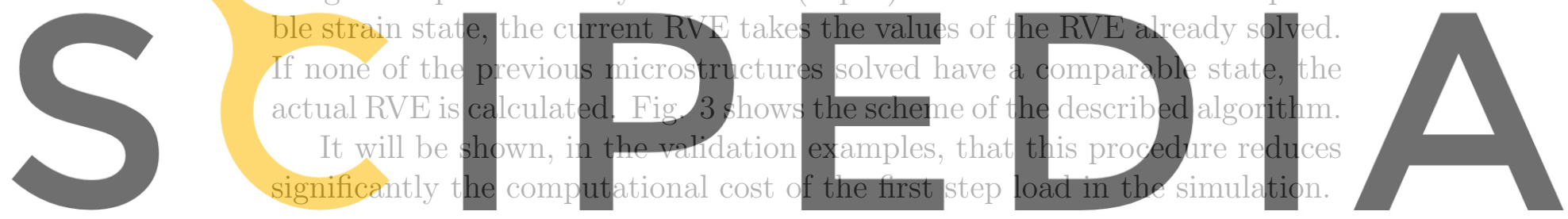

Register for free at https//Www. Sclpedia.com to download the version without the watermark

The proposed NLS has been implemented in PLCd [41], a parallel finite

element code that works with 3D solid geometries. The PLCd code has al-

ready implemented the $\mathrm{FE}^{2}$ procedure [10]. In the code, a Newton-Raphson scheme is adopted to solve the non-linear problem. To facilitate the convergence of the whole problem, the tangent constitutive tensor at the integration point is necessary to obtain the global tangent stiffness matrix.

A perturbation method is used to obtain a numerical approximation of the homogenized tangent constitutive tensor of the RVE in the integration point. The method implemented is analogous to the one proposed by Martinez et al. in [42] (see AppendixB). Being the only difference that in current procedure the perturbations must be applied on the RVE instead of applying them to a constitutive equation. The $n$ small perturbations $\left(\delta \bar{\varepsilon}_{j}\right)$ are applied to the homogenized or structural strain vector. The RVE is solved $n$ times and as result gives the $n$ stress vector $\delta^{j} \overline{\boldsymbol{\sigma}}$. Therefore, the $j$ columns of the tangent constitutive tensor for the RVE can be obtained by the following equation: 


$$
\overline{\boldsymbol{C}}_{j}^{t} \equiv \frac{\delta^{j} \overline{\boldsymbol{\sigma}}}{\delta \overline{\boldsymbol{\varepsilon}}_{j}}
$$

The calculation of the tangent stiffness tensor is necessary to obtain a good convergence of the problem but it is computationally expensive. This shows again the necessity to reduce the number of times in which this calculation is performed, and proves the necessity of having a non-linear strategy to conduct the simulation.

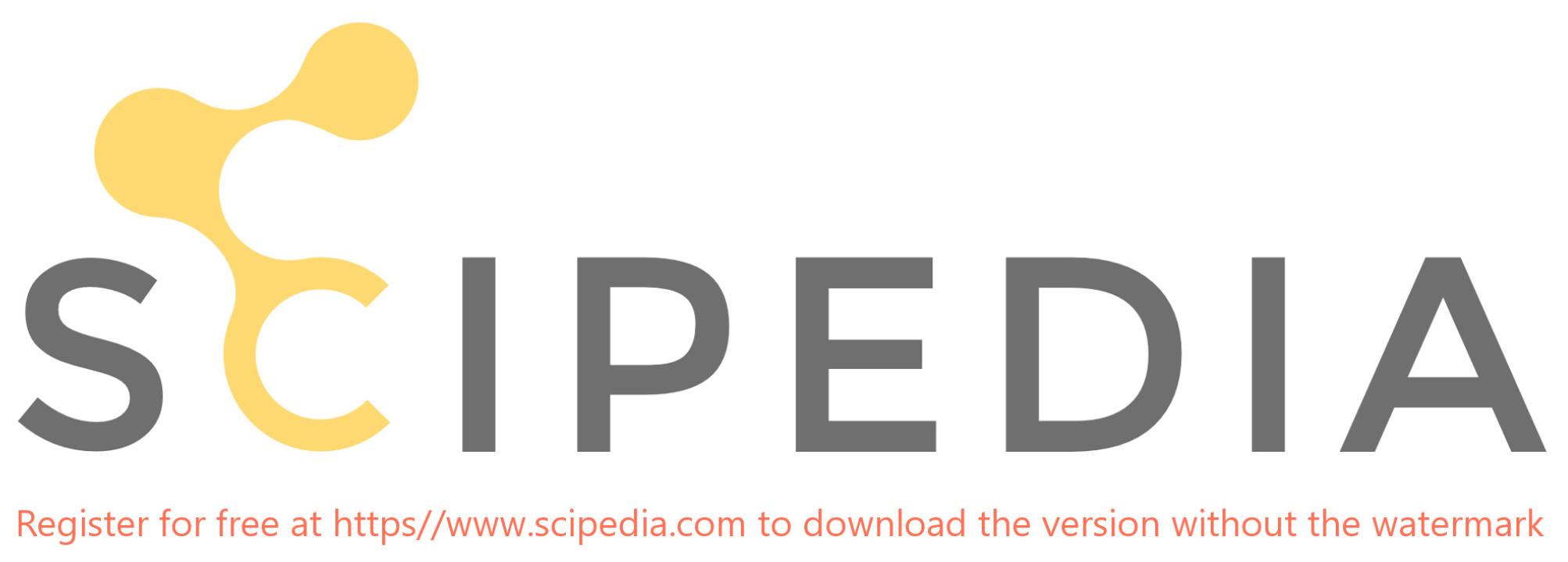




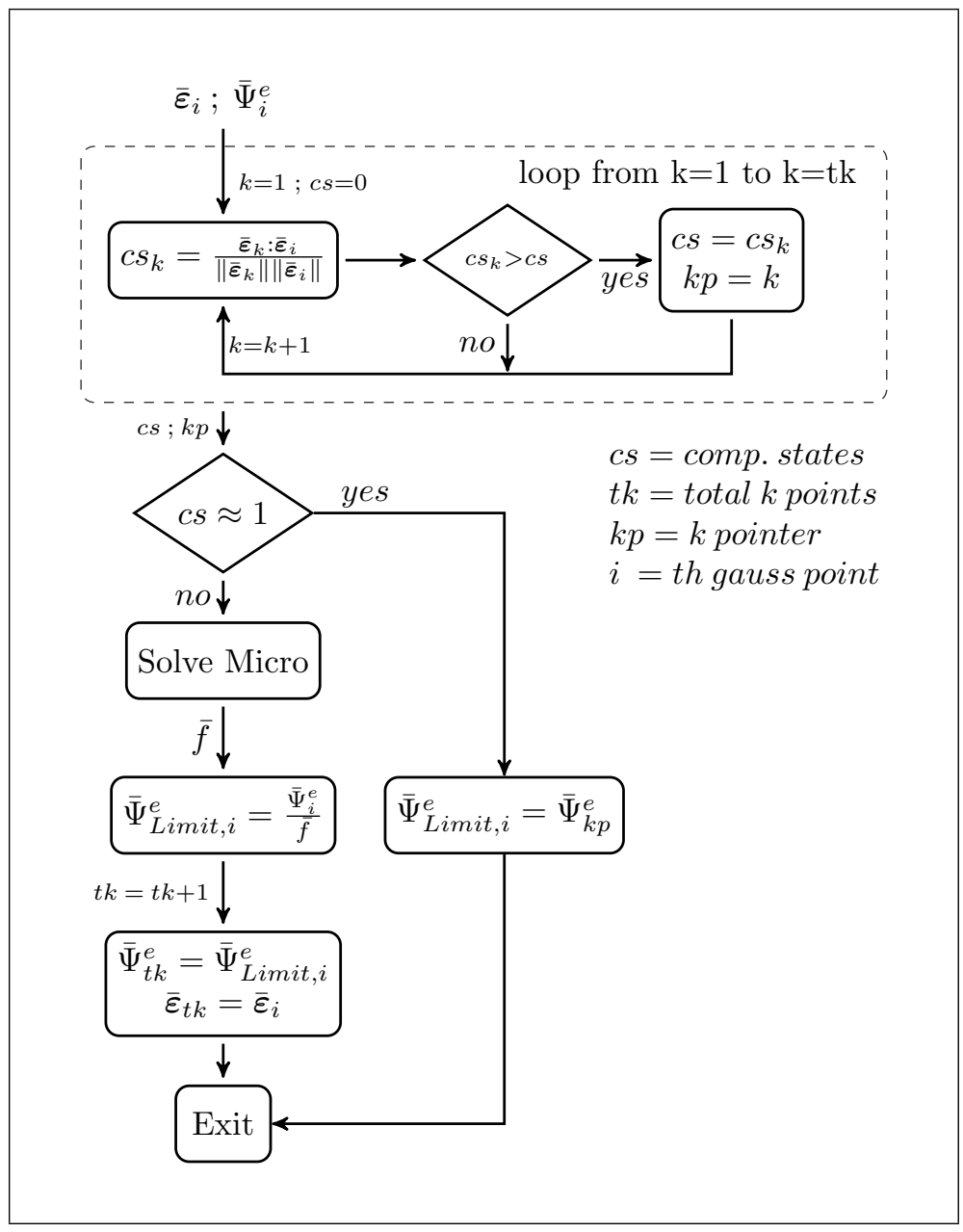

Figure 3: Smart first step algorithm scheme. 


\section{Energy dissipation in a multi-scale analysis}

The solution of material non-linear problems with a numerical double scale homogenization procedure not only should be affordable computationally, but the results obtained in the non-linear process must be also correct. Therefore, the procedure must dissipate the same energy in both scales. In order to conserve the dissipated energy through the scales, the following methodology is proposed.

\subsection{Fracture energy}

Fracture mechanics presents the fracture energy per unit of area, $G_{f}$, as a property of the material. This energy can be calculated as:

$$
G_{f}=\frac{W_{f}}{A_{f}}
$$

where, $W_{f}$ is the energy dissipated by the fracture at the end of a quasi-static process, and $A_{f}$ is the total fractured area. This fracture energy is the link between the fracture mechanics and the constitutive model based on classical solid mechanics. The constitutive model must satisfy:

1. The good representation of behavior of a set of points inside of a finite domain.

2. The same energy dissipated by the total volume as the one dissipated by the solid in the real fracture process.

Considering a simple tensile test, the constitutive model must verify the following condition of dissipation:

$$
\underbrace{W_{f}=G_{f} A_{f}}_{\text {Fracture Mechanics }} \equiv \underbrace{\Pi^{d}=\int_{v} g_{f} d V}_{\text {Solid Mechanics }}
$$

where $g_{f}$ is the maximum specific energy dissipated by the constitutive model. Eq. (8) states that the energy delivered to the tensile test must be equal to the energy dissipated by the constitutive model. In solid mechanics, the dissipation phenomena is located in a volume that can be represented as $V_{d}=A_{f} L_{f}$, where $L_{f}$ is a fracture length. For FE method the localization phenomena in one strip of finite elements is sought, therefore $L_{f}$ is commonly approximated by some reference length of the finite element. This length is a 
parameter that accounts for the amount of energy dissipated by the fractured material. Replacing the volume $V_{d}$ in Eq. (8) the following expression is obtained:

$$
W_{f}=G_{f} A_{f}=g_{f} A_{f} L_{f}
$$

From Eq. (9) the relation between the material parameter $G_{f}$ and the specific energy dissipated $g_{f}$ is found:

$$
g_{f}=\frac{G_{f}}{L_{f}}
$$

\subsection{Localization at the microstructural scale}

In multi-scale procedure, the specific energy at the macro structural scale is obtained by the following equation:

$$
g_{f}=\frac{1}{V_{m}} \int_{\Omega_{m}} g_{f}^{m} d V_{m}
$$

where the index $m$ is used to reference the microstructural scale variables.

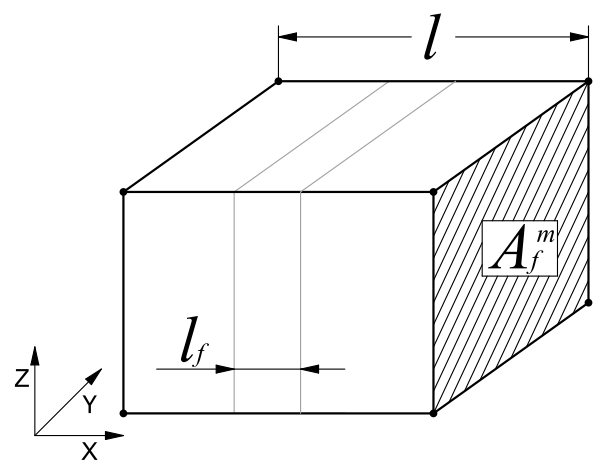

Figure 4: Representative volume of the subscale.

Taking the same consideration than macroscale solid behavior, now the dissipation phenomena is located at microstructural level (see Fig. 4). In such case, we have the following dissipated equation:

$$
g_{f}=\frac{1}{A_{f}^{m} l} g_{f}^{m} A_{f}^{m} l_{f}
$$


where, $A_{f}^{m}$ is the RVE cross section area, $l$ is the length in normal direction of $A_{f}^{m}$ and $l_{f}$ is the fracture length at the microstructure (RVE). With Eq. (12) is possible to obtain the specific energy $g_{f}^{m}$ dissipated at the microstructural scale level as:

$$
g_{f}^{m}=\frac{g_{f} l}{l_{f}}=\frac{G_{f} l}{L_{f} l_{f}}
$$

Eq. (13) shows the relationship between the $g_{f}^{m}$ and $G_{f}$ which ensures to dissipate the same energy by the solid mechanics, using a multi-scale method, than the one obtains with a tensile test. The validity of this relation is proved in the following example.

\subsection{Validation example}

A simple tensile numerical test over a material sample is simulated. The objective of this example is to analyze the objectivity of the response obtained using the proposed $\mathrm{FE}^{2}$ method. The same test using a classical one scale FE method is also solved for comparison purposes. The geometry, the supports and the displacements scheme of the simulated structure is presented in Fig. 5. The applied fixed displacement is represented by the arrows in the figure.

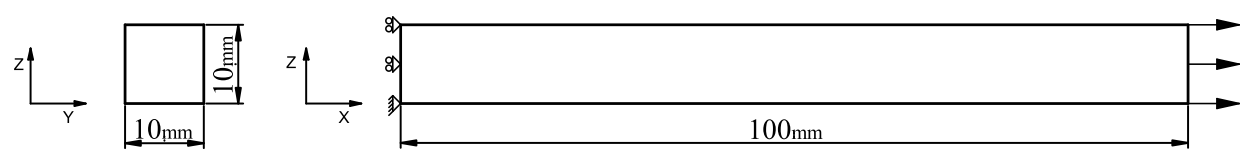

Figure 5: Structure simulated in the tensile test.

\subsubsection{Material}

The simple material used in the tensile test takes the properties shown in Table 1. The constitutive model chosen is an explicit scalar damage model with exponential softening $[43,44]$. For this particular case, where the stress state is uniform and there is only one simple material, and in order to help the localization of the softening problem, the elastic limit is increased in some elements (drawn with gray color in Fig. 6) up to a value of $\sigma_{\text {Limit }}=102 \mathrm{MPa}$.

\subsubsection{FE meshes}

The finite element employed to solve the problem is a first order hexahedra element. The example is solved for different combinations of finite element 


\begin{tabular}{lllll}
\hline Properties & $E[\mathrm{GPa}]$ & $\nu[-]$ & $\sigma_{\text {Limit }}[\mathrm{MPa}]$ & $G_{f}\left[\mathrm{~kJ} / \mathrm{m}^{2}\right]$ \\
\hline Values & 100 & 0 & 100 & 20 \\
\hline
\end{tabular}

Table 1: Simple material properties used in the tensile test.

meshes. Fig. 6 shows the different mesh sizes used in the simulation. The left side of Fig. 6 shows the three different meshes used for the structural scale. The mesh Macro1 has 10 finite elements, the mesh Macro2 has 84 elements and finally the mesh Macro3 has 656 elements. On the other hand, the right side of Fig. 6 shows the two different meshes used for the microstructural scale. The mesh Micro1 has 125 finite elements and the mesh Micro2 has 729 elements.
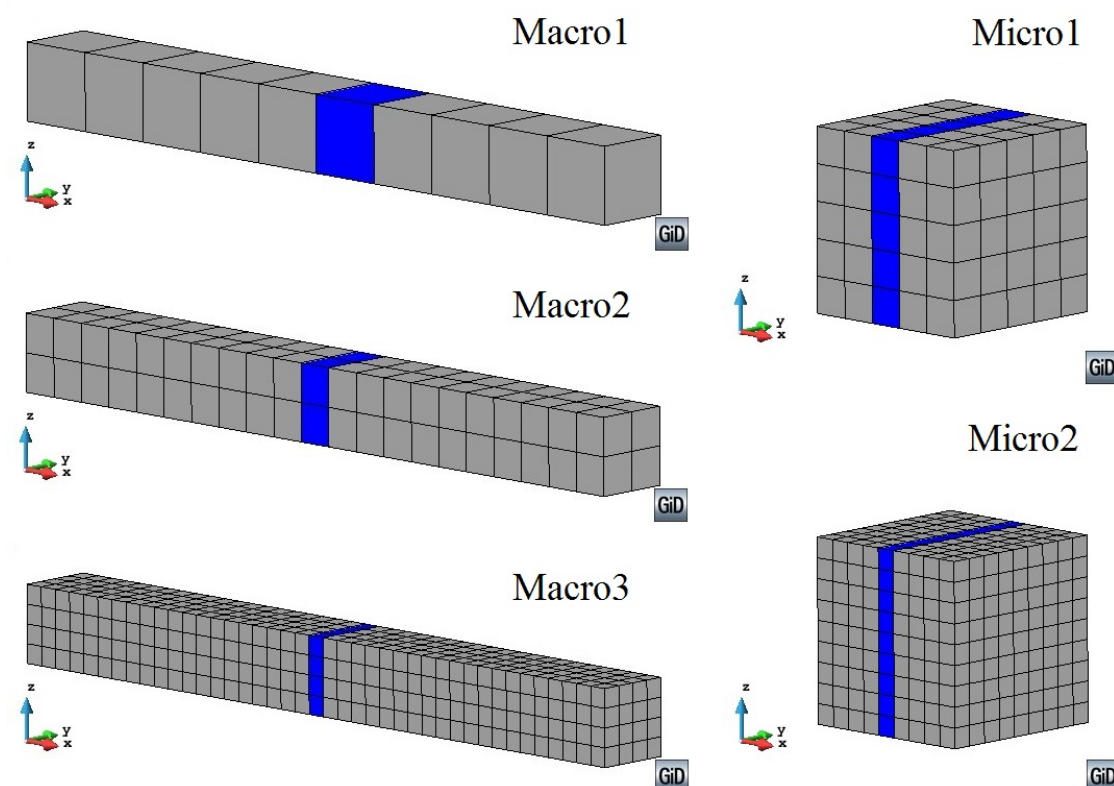

Figure 6: Different meshes used in the tensile test.

\subsubsection{Results}

The results obtained with the different mesh combinations are shown graphically in the Fig. 7. As can be observed from the figure, the results are equal for all combinations, and for both methods.

For the case considered, it is possible to validate the numerical results with analytical calculations, knowing the area of the specimen, the Young's 


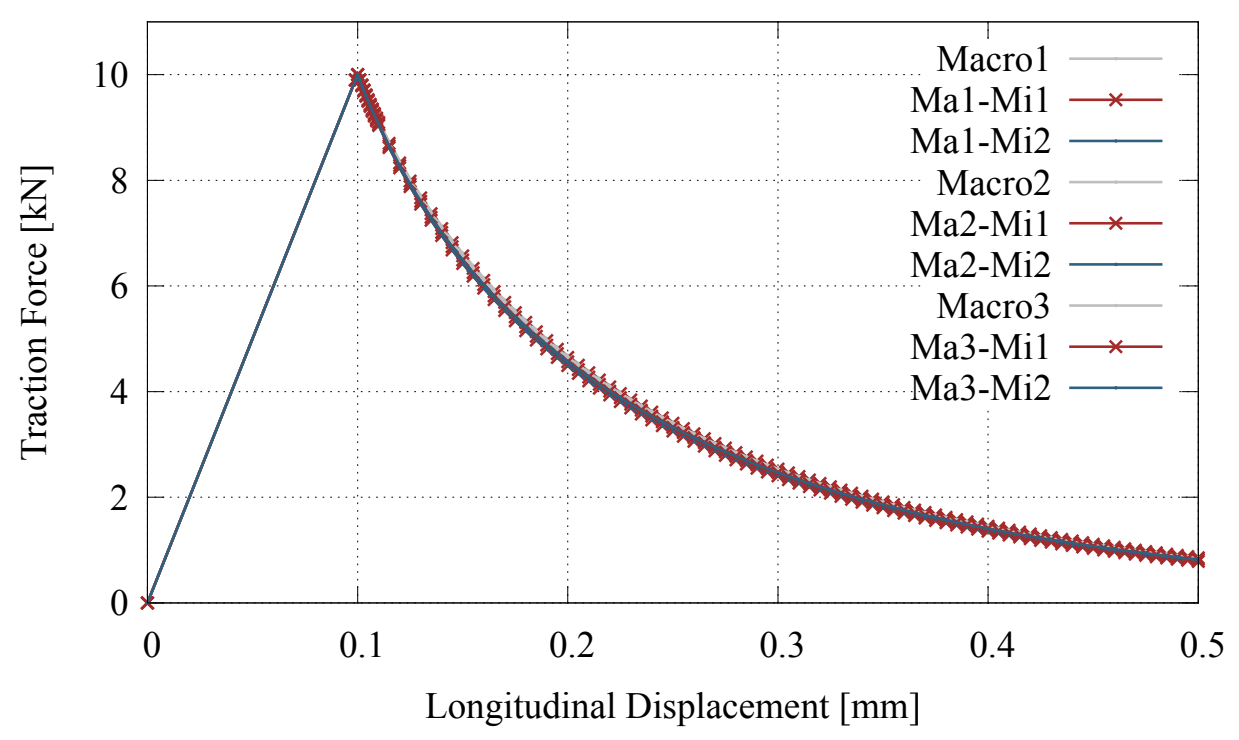

Figure 7: Traction force vs displacement curves obtained in the tensile test.

modulus and the maximum tensile stress that can be applied, the maximum load and displacement in the beam is:

$$
\begin{aligned}
& F_{\text {max }}=\sigma_{\text {Limit }} \cdot A=10 \mathrm{kPa} \\
& d_{F_{\text {max }}}=\frac{L \cdot \sigma_{\text {Limit }}}{E}=0.1 \mathrm{~mm}
\end{aligned}
$$

It is also possible to calculate analytically the dissipated energy at the end of the test:

$$
W_{f}=G_{f} \cdot A=2 \mathrm{~J}
$$

If this energy is calculated from the numerical models, the following table is obtained:

\begin{tabular}{llll}
\hline Energy $[\mathrm{J}]$ & Macro1 & Macro2 & Macro3 \\
\hline One scale & 1.728 & 1.737 & 1.748 \\
Micro1 & 1.752 & 1.741 & 1.777 \\
Micro2 & 1.713 & 1.761 & 1.812 \\
\hline
\end{tabular}

Table 2: Dissipated energies obtained in the tensile test.

The difference between the estimated value and the ones show in Table 2 
is because at the numerical analysis the simulation has been stopped at $0.5 \mathrm{~mm}$. It has to be noted also that the dissipation obtained with all mesh configurations is practically the same. Which proves the consistency of the formulation proposed.

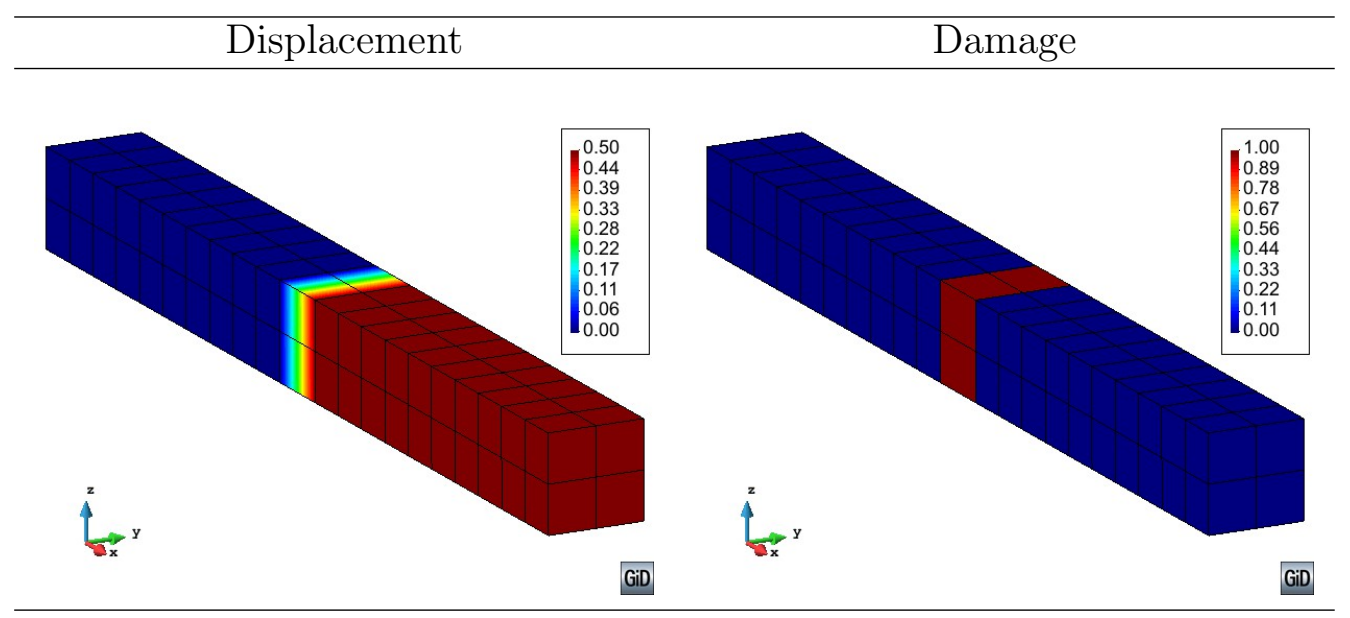

Figure 8: Macrostructural results obtained at the end of the tensile test.

The localization of non-linear phenomena in one strip of finite elements at the structural scale is shown in the Fig. 8, for the analysis made with Macro2 mesh. This figure shows that damage is concentrated in the central zone of the material sample, and therefore, the displacement too. In the proposed multi-scale method the localization phenomena must be observed also at the microstructural scale. As an example, Fig. 9 shows the microstructural displacement and damage obtained at the end of one tensile numerical test. In the figure can be observed that both results are localized in one strip of finite elements in the RVE meshes. 


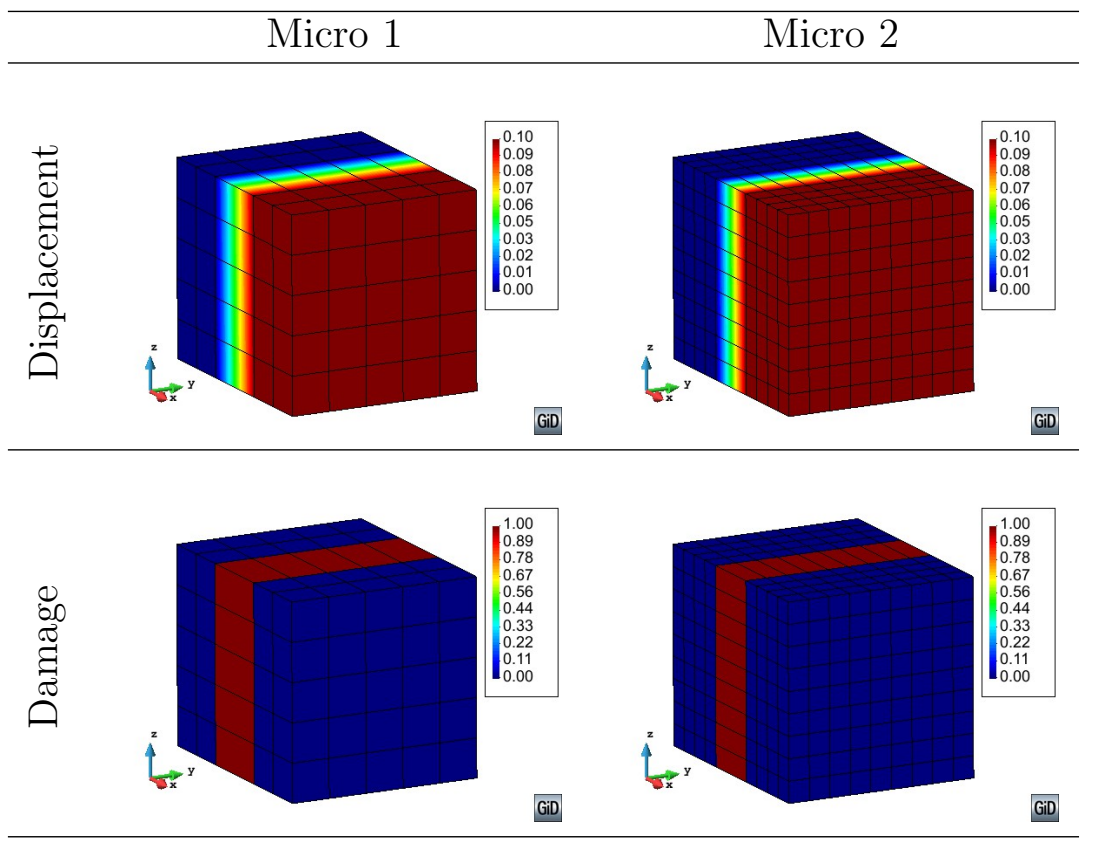

Figure 9: Microstructural results obtained at the end of the tensile test. 


\section{Numerical examples of non-linear analyses}

\subsection{Tensile test of a plate with a hole.}

The objective of this example is to show the performance of the NLS developed, as well as to analyze the failure of the structure localizes in a strip of elements. The test is a tensile test made on a plate with a hole in its center. Due to the symmetry of the geometry and of the load applied, only a quart of the real structure is simulated. Fig. 10 shows the modeled geometry, the supports and the displacements scheme in the numerical model. The applied fixed displacement is represented by the arrows in Fig. 10.

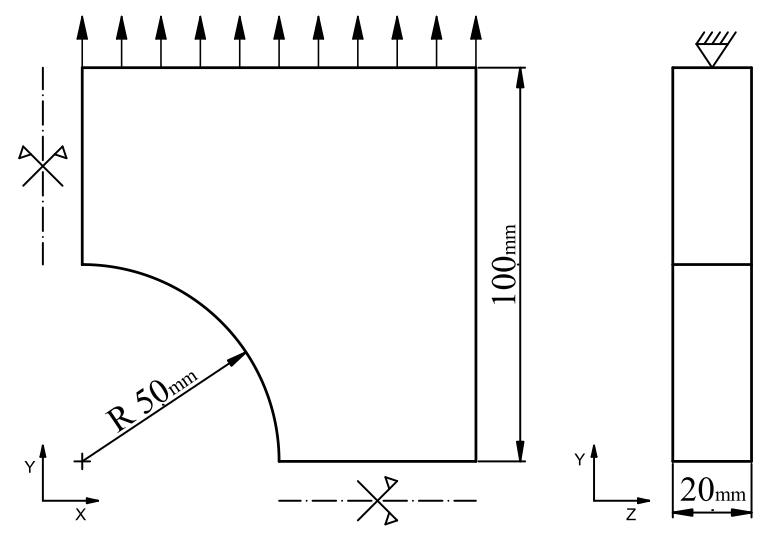

Figure 10: Simulated structure of the plate with a hole.

\subsubsection{Material}

Table 3 shows the properties of the simple material used. The constitutive model of the material is the same (explicit scalar damage) that has been used in the previous validation example 3.3.

\begin{tabular}{lllll}
\hline Properties & $E[\mathrm{GPa}]$ & $\nu[-]$ & $\sigma_{\text {Limit }}[\mathrm{MPa}]$ & $G_{f}\left[\mathrm{~kJ} / \mathrm{m}^{2}\right]$ \\
\hline Values & 100 & 0.15 & 100 & 10 \\
\hline
\end{tabular}

Table 3: Simple material properties used in the plate with a hole. 


\subsubsection{FE meshes}

To analyze the response's objectivity in the test, two finite element meshes have been used for the macroscopic model. Fig. 11 shows the mesh sizes employed. Mesh1 has 360 finite elements while Mesh2 is more dense and has 2880 elements. The microstructural model and finite element meshes are the same than the ones used in previous validation example 3.3.

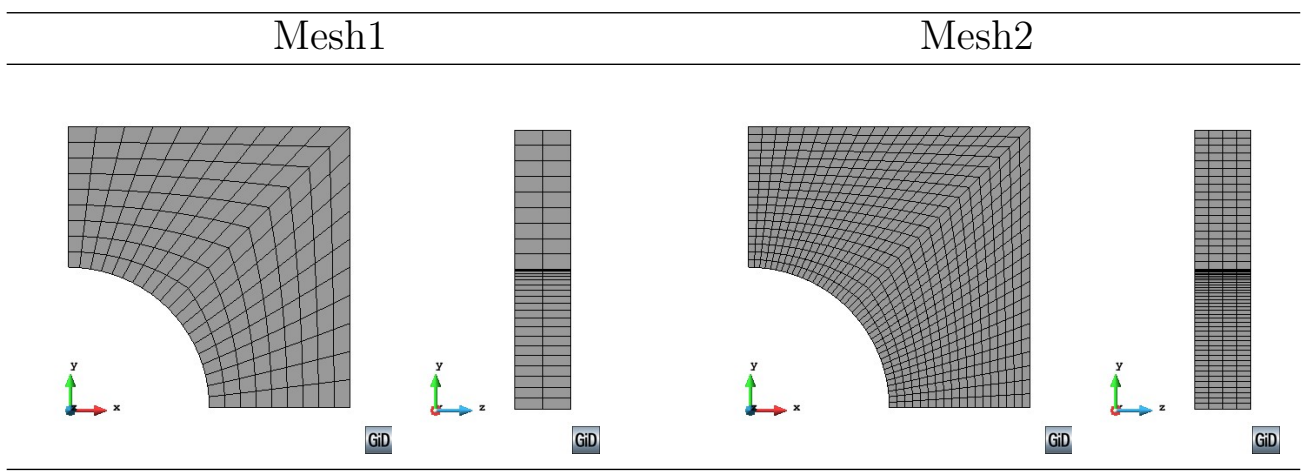

Figure 11: Different meshes used in the plate with a hole.

\subsubsection{Results}

Fig. 12 shows the traction force vs displacement curves obtained for the different mesh combinations. This figure shows that the results are almost equal. Therefore, the result obtained with the proposal method is mesh independent. The curves show than the maximum force does not pass of $80 \mathrm{kPa}$ and it is obtained for an applied displacement of $0.08 \mathrm{~mm}$.

The dissipated energy for the different mesh configurations used in this example is shown in Table 4 . From the Table 4 can be observed than the worst difference between two results is less than $2 \%$.

\begin{tabular}{lll}
\hline Energy $[\mathrm{J}]$ & Mesh1 & Mesh2 \\
\hline Micro1 & 3.152 & 3.135 \\
Micro2 & 3.192 & 3.169 \\
\hline
\end{tabular}

Table 4: Dissipated energy in the plate with a hole.

To fully understand the behavior of the structure under the applied load several figures for different load state are presented. Fig. 13 and Fig. 14 show the results obtained for Mesh1 and Mesh2, respectively. In the figures, 


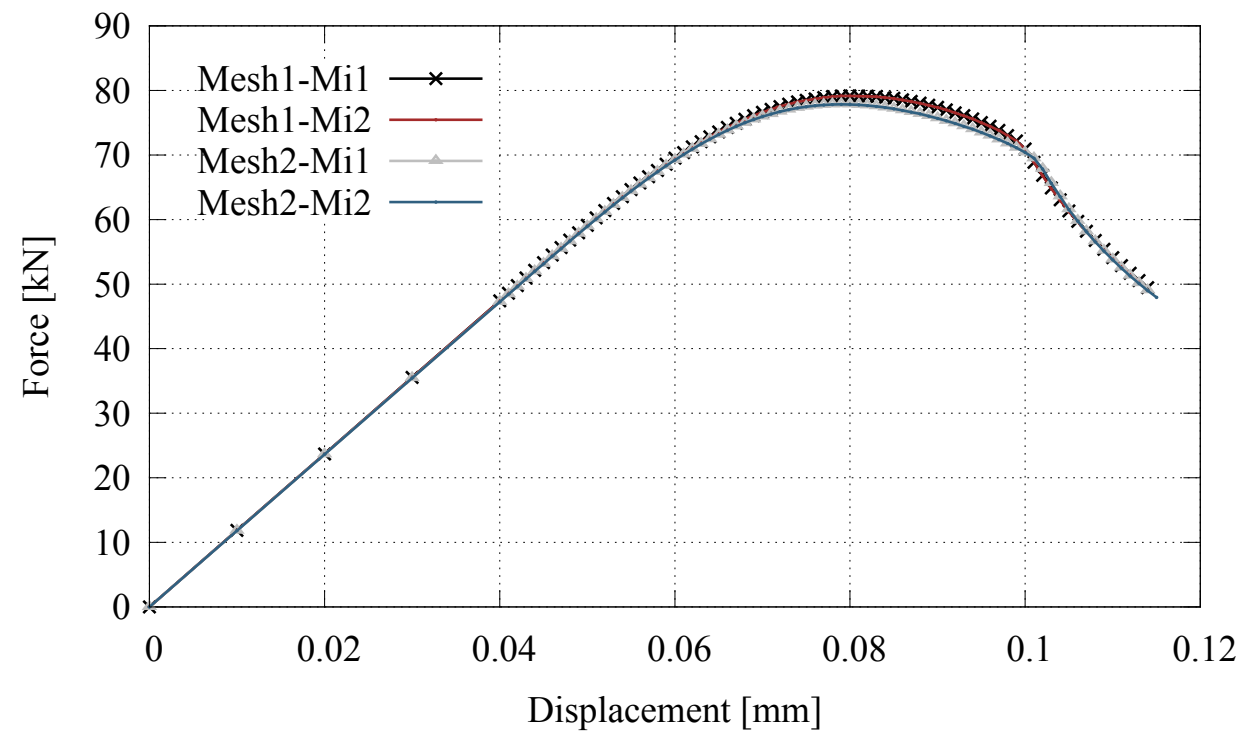

Figure 12: Forve vs Displacement for the plate with a hole.

strain and stress in $\mathrm{Y}$ direction and scalar damage are presented for four different fixed displacement steps.

The figures show how at the beginning of the test (label a) $d=0.05 \mathrm{~mm}$ ), the maximum strain and stress are located at the inner border of the hole. Then, the non-linear process starts there and, as a consequence, the damage increases in that zone. Due to constitutive model used, when the damage increases in the material the stress decreases. As the applied displacement continues increasing, the structure transfers the load to non damaged zones. Therefore, the zone with maximum stress moves from the inner border to the central part and the strain and damage move on as a constitutive response. At the end of the test (label $\mathrm{d}$ ) $\mathrm{d}=0.11 \mathrm{~mm}$ in the figures), the maximum stress is located in the right external border of the plate. It is important to mention that during the test, the stress in the structure never takes values over the limit imposed (see Table 3) as can be observed in Figs. 13 and 14. Finally, the figures show how the model is capable of localizing all damage in a single strip of finite elements. 

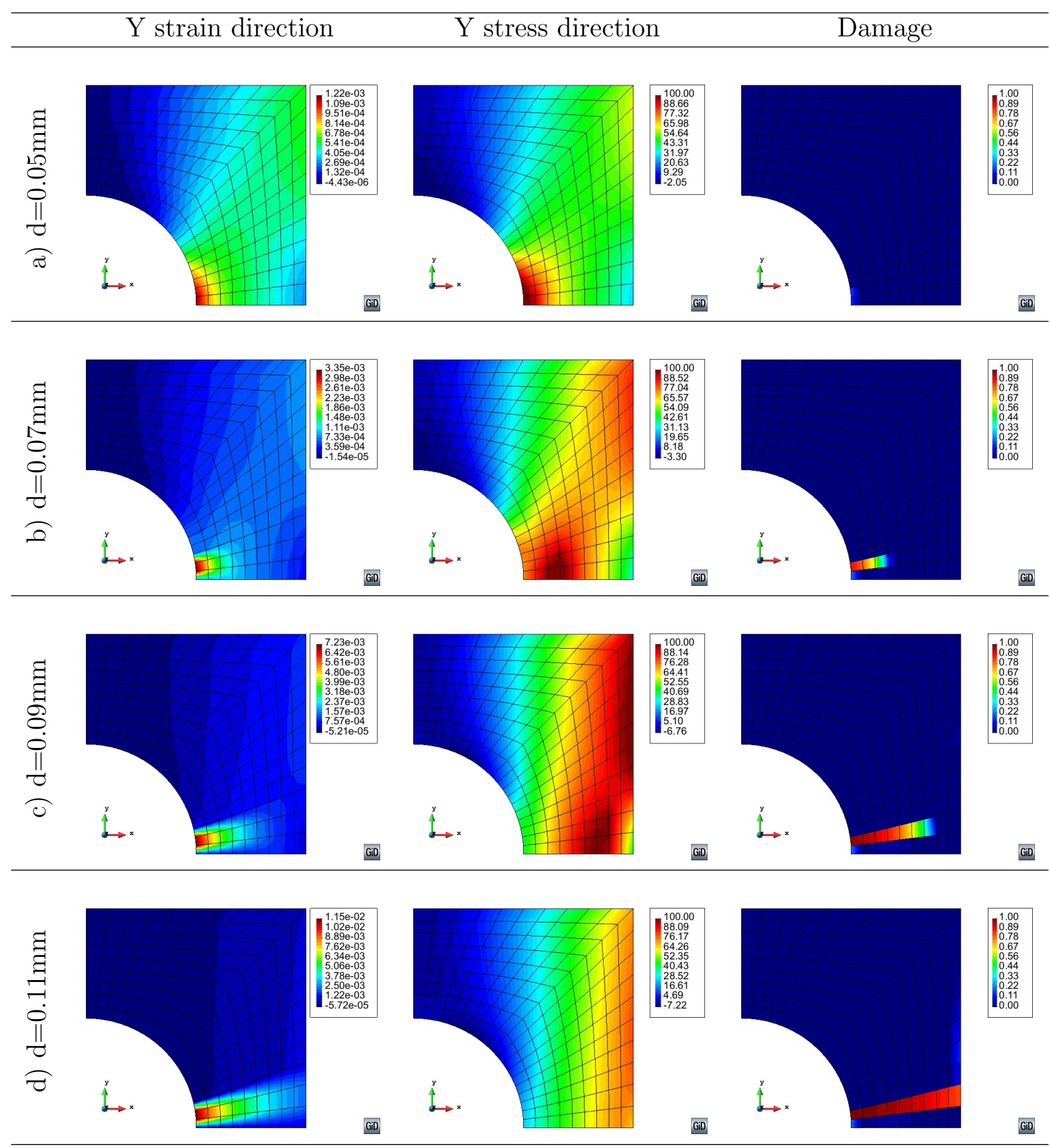

Figure 13: Results obtained in the plate with a hole to Mesh1. 

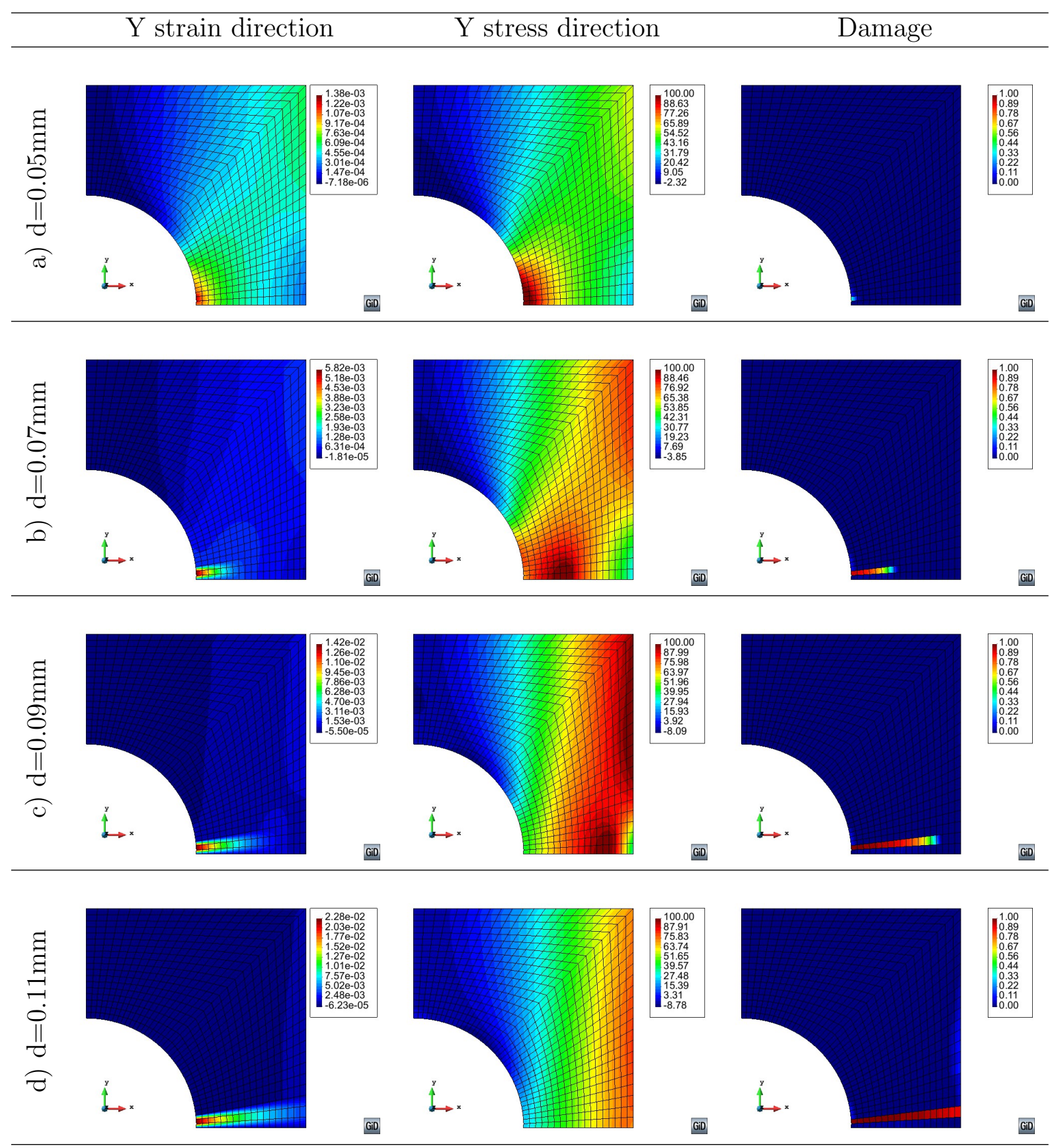

Figure 14: Results obtained in the plate with a hole to Mesh2. 


\subsubsection{Computational times}

To show the advantage of using the NLS proposed, in comparison with a full classical $\mathrm{FE}^{2}$, the calculation times are presented herein. A full $\mathrm{FE}^{2}$ solves the microstructural problem for every integration point of the structure, and for every time step. This procedure does not distinguish between linear range and non-linear range.

Tables 5 and 6 present the calculation times required to solve the shown example in the same desktop computer, an Intel ${ }^{\circledR}$ Core $^{\mathrm{TM}}$ i7-2600 CPU @ $3.40 \mathrm{GHz}$ with $8 \mathrm{~GB}$ of RAM. The tables show the times used by the $\mathrm{FE}^{2}$ and by the $\mathrm{FE}^{2}$ with the NLS incorporated. The speed ratio column has the relation times between both methodologies.

Table 5 has the total real times necessary to complete the numerical test, up to $\mathrm{d}=0.115 \mathrm{~mm}$, for all mesh configurations. The speed ratio variable shows that the advantage of using the developed strategies increases specially when the size of the macrostructure's mesh increases. This is an expected result because, in larger meshes, the proportion between linear and non-linear elements becomes also larger.

\begin{tabular}{lccl}
\hline Model & \multicolumn{1}{c}{$\mathrm{FE}^{2}$} & $\mathrm{FE}^{2}+\mathrm{NLS}$ & Speed ratio \\
\hline Mesh1-Micro1 & $1: 21: 53$ & $0: 28: 19$ & 2.89 \\
Mesh1-Micro2 & $8: 41: 19$ & $3: 10: 44$ & 2.73 \\
Mesh2-Micro1 & $11: 19: 49$ & $2: 29: 28$ & 4.55 \\
Mesh2-Micro2 & $76: 40: 33$ & $18: 39: 33$ & 4.11 \\
\hline
\end{tabular}

Table 5: Computation times requested to solve the plate with a hole [hs:min:seg].

On the other hand, it is important to mention that when a RVE becomes non-linear, its computational cost is more expensive than when it is linear. This is because, besides the possible iteration required by the RVE to obtain the correct non-linear solution, the estimation of the tangent constitutive tensor by perturbation method requires to solve the RVE six more times (see Subsection 2.3).

Consequently, when the number of non-linear elements in a problem increase, the efficiency of the proposed method decreases. For the analyzed example, if the simulation is stopped at the maximum admissible force in the structure (around $d=0.08 \mathrm{~mm}$ in Fig. 12) which probably the most interesting value for an engineer, the speed ratio would be better. To prove this, let's consider the Mesh2-Micro2 simulation. In this case, when the maximum 
load is applied $(\mathrm{d}=0.08 \mathrm{~mm})$ there are only 392 elements in non-linear range, instead of 576 elements for $\mathrm{d}=0.115 \mathrm{~mm}$, and therefore at this load step the speed ratio is of 7 instead of 4.11 .

This simulation is also used to validate the effect of the Smart First Step procedure. To do so, Table 6 shows the computational times consumed for the first step in each one of the simulations conducted. The times shown prove that using SFS strategy improves highly the computational efficiency also for small mesh sizes, as speed ratio variable shows. The table also shows that the number of RVE solved by the SFS is independent of the mesh used in the microstructural problem.

\begin{tabular}{l|rc|cc|l}
\hline \multirow{2}{*}{ Model } & \multicolumn{2}{|c|}{ without SFS } & \multicolumn{2}{c|}{ with SFS } & \multirow{2}{*}{ Speed } \\
\cline { 2 - 5 } & \multicolumn{1}{|c|}{ Time } & RVE solved & Time & RVE solved & ratio \\
\hline Mesh1-Micro1 & $0: 17.9$ & 2880 & $0: 01.0$ & 151 & 17.9 \\
Mesh1-Micro2 & $1: 48.3$ & 2880 & $0: 06.5$ & 151 & 16.7 \\
Mesh2-Micro1 & $2: 12.0$ & 23040 & $0: 02.6$ & 303 & 50.8 \\
Mesh2-Micro2 & $14: 05.9$ & 23040 & $0: 12.5$ & 303 & 67.7 \\
\hline
\end{tabular}

Table 6: First step computation times in the plate with a hole [min:seg]. 


\subsection{Industrial Component}

In order to validate the efficiency obtained with the NLS when it is applied to the solution of a real structure, in the following is included the non-linear simulation of an structural component. In this case, the structure selected for analysis is the industrial component show in Fig. 15. The geometry of this engine stiffener has been proposed in the framework of M-RECT Project. The stiffener is linked on one side to the gearbox, and on the other side to the engine. This component has the objective of improving the connection between engine and gearbox, as well as changing the dynamic properties of the overall structure.
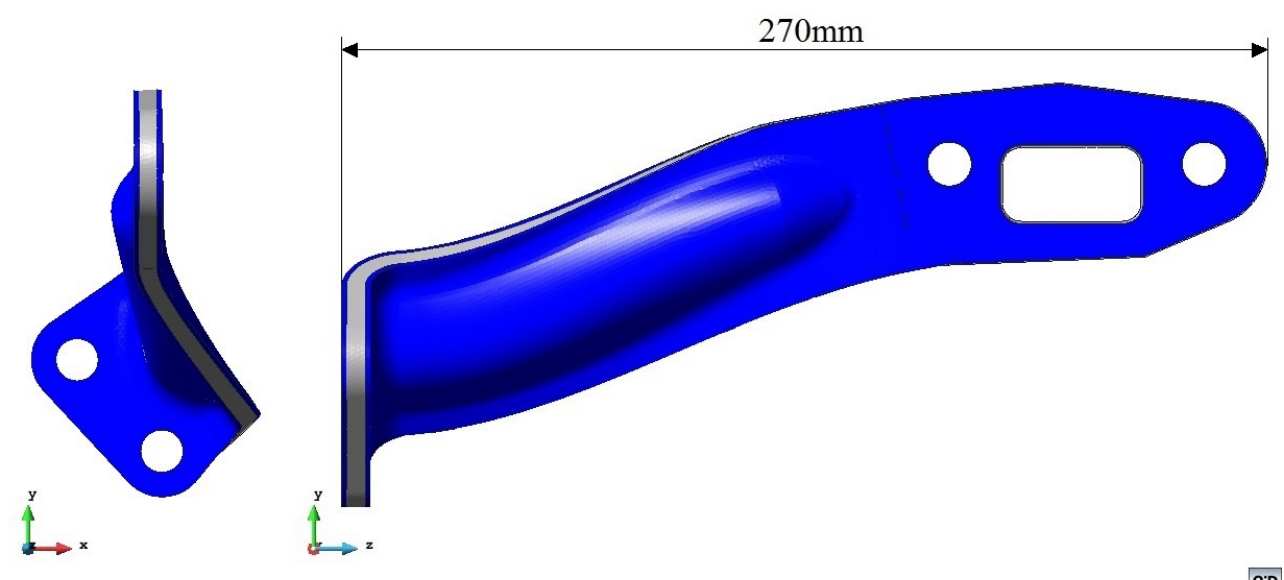

Figure 15: Engine stiffener part.

\subsubsection{Materials}

The material that will be used for the stiffener, different from the one used in M-RECT, is a laminated thermoplastic composite. Therefore, the material properties vary through the laminate thickness and respect to the laminate's reference direction. The composite is made with three orthotropic sheets (see Fig. 15). The two external sheets (drawn in blue) have a thickness of $1.5 \mathrm{~mm}$ each one and, the core sheet (drawn in gray) has a thickness of $5.5 \mathrm{~mm}$.

The external laminae is composed by carbon fibers in an epoxy matrix. The periodic microstructure of the external sheets can be represented by the RVE shown in the Fig. 16. The laminate has a $40 \%$ of cylindrical long fiber volume. 


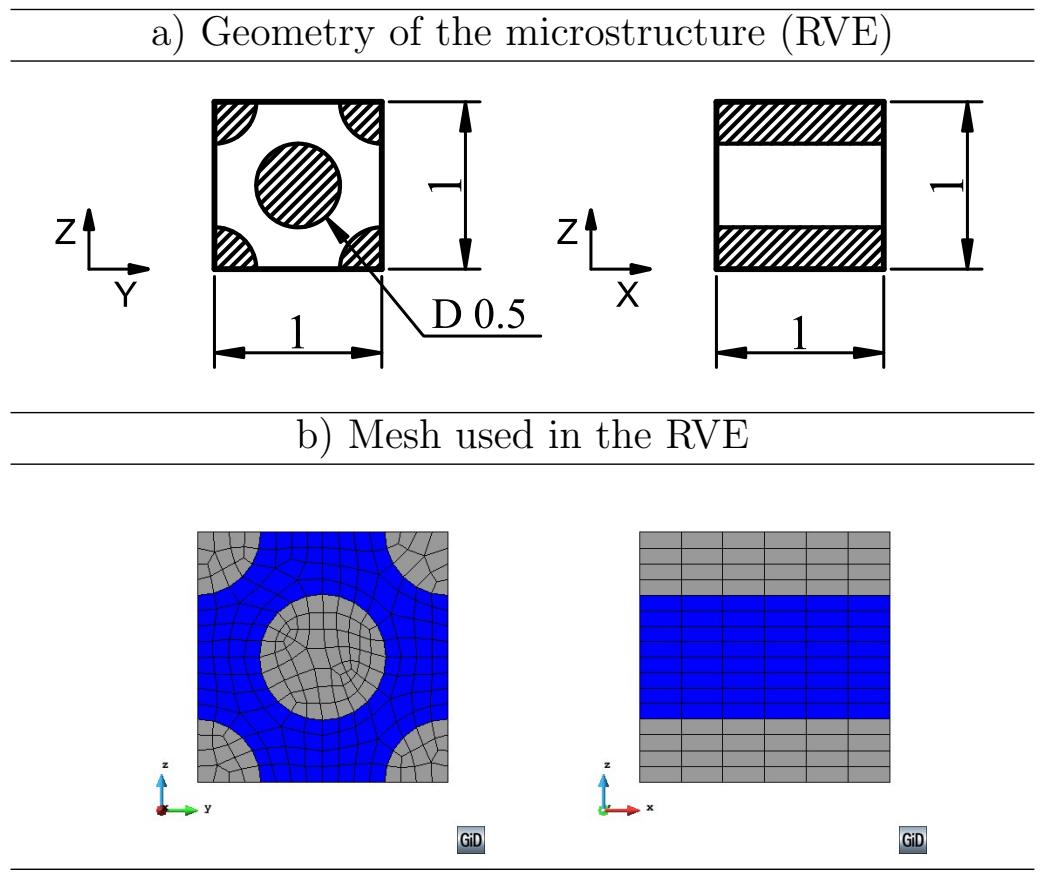

Figure 16: Geometry and mesh of the RVE used in the external sheets.

The matrix is an isotropic material, simulated with an explicit scalar damage constitutive model with exponential softening (resin epoxy HSC Epikote 4652). The long fiber is modeled with an elastic constitutive model (Grafil TR30S 3K carbon fiber). The properties of these simple materials are shown in Table 7 [9].

\begin{tabular}{lllll}
\hline Material & $E[\mathrm{GPa}]$ & $\nu[-]$ & $\sigma_{\text {Limit }}[\mathrm{MPa}]$ & $G_{f}\left[\mathrm{~J} / \mathrm{m}^{2}\right]$ \\
\hline Epoxy matrix & 4.52 & 0.36 & 68 & 780 \\
Carbon fiber & 235 & 0.21 & 4410 & - \\
\hline
\end{tabular}

Table 7: Simple material properties from Perez et al. (2013).

Finally, the FE mesh employed to analyze the RVE is shown in Fig. 16. The mesh uses 1464 first order hexahedra finite elements.

On the other hand, the core sheet of the engine stiffener is a TenCate commercial product, Cetex TC1200 PEEK 5HS LAMINATE. The properties of this material have been obtained from TenCate website [45] and are shown in Table 8. For the simulation, the core material is modeled using an elastic constitutive model. 


\begin{tabular}{llll}
\hline Propertie $[\mathrm{GPa}]$ & $E\left(0^{\circ}\right)$ & $E\left(90^{\circ}\right)$ & $G($ In plane $)$ \\
\hline TenCate lamina & 56.1 & 55.6 & 4.5 \\
\hline
\end{tabular}

Table 8: TenCate Lamina properties.

\subsubsection{Mesh and boundary conditions}

Fig. 17 label a) shows the mesh used to simulate the engine stiffener. The mesh has 355.302 first order tetrahedra finite elements. The external laminae requires 108.041 elements while the core lamina has 247.261 elements.

a) Mesh used in the engine stiffener

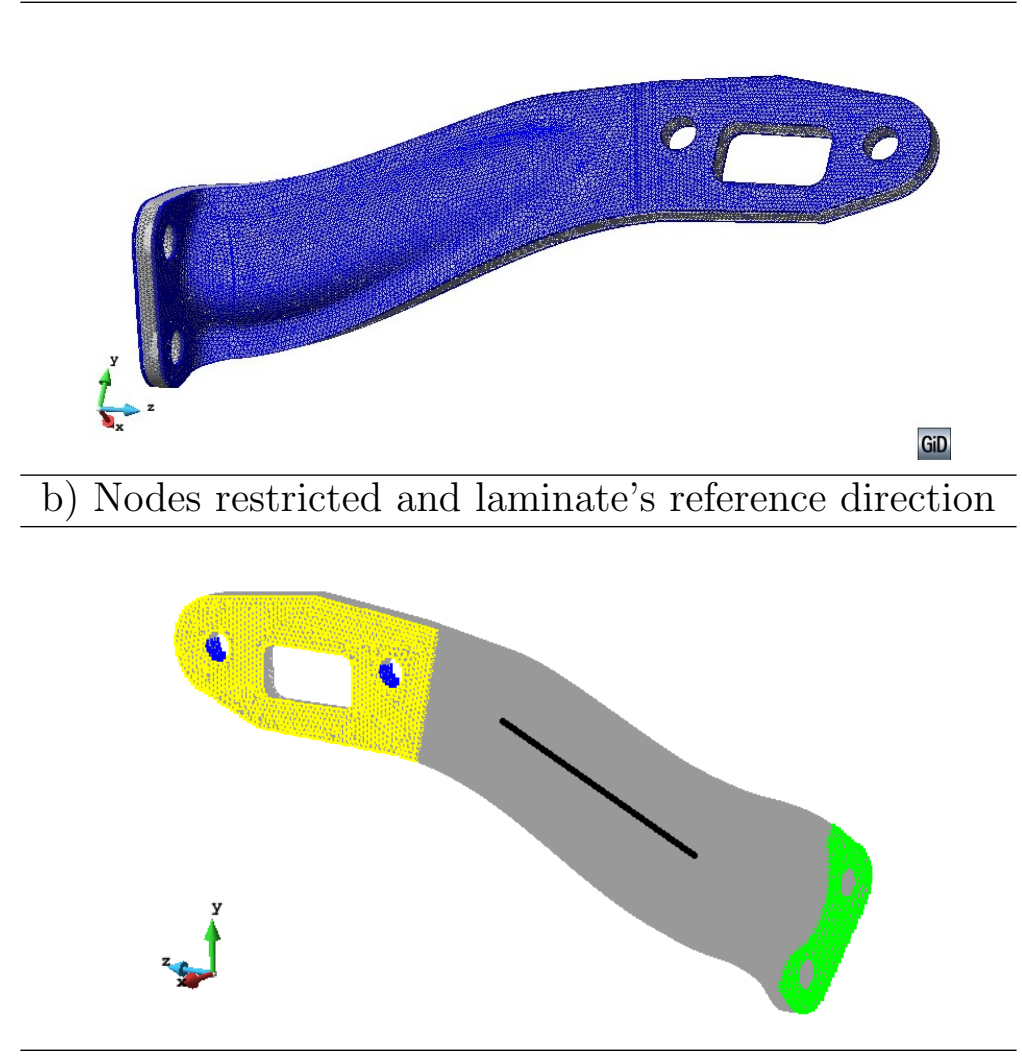

Figure 17: Mesh and boundary conditions used in the engine stiffener.

The nodes that will be restricted and the laminate's reference direction are shown in Fig. 17 label b). The nodes with green color are over the face in contact with the gearbox. These nodes have a zero movement restriction in all directions. On the other hand, the nodes drawn in yellow are on the 
face in contact with the engine. In this case, the restriction on these nodes is a fixed displacement in X direction. The laminate's reference direction is the long fiber longitudinal direction in the external laminae.

\subsubsection{Results}

The analysis conducted on the first step to evaluate the elastic energy density available in each integration point allows defining a "possible damage" map of the structure, as it is shown in Fig. 18 label a), where $\bar{f}$ is presented. The blue zones in the external sheets have a $\bar{f}$ near to zero and then, these zones will have a $\bar{\Psi}_{\text {Limit }}^{e}$ high while the zone with a $\bar{f}$ near to 1 (critical zones) will have a smaller elastic energy density available. Therefore, these zones are the regions where non-linear process has more possibilities to start. In this example, the SFS has required analyzing 6.514 RVEs to determine the threshold functions of the whole structure. This quantity represents only the $6 \%$ of the elements on the external laminae.

The numerical simulation has been stopped to a fixed $\mathrm{X}$ displacement of $1.36 \mathrm{~mm}$. The homogenized stress at the end of the analysis in the laminate's reference direction is shown in Fig. 18 label b). From the figure it is observed that the maximum absolute stress is a compressive stress and it is located near to the face in contact with the gearbox. The maximum tensile stress is located in the same region but in the opposite external sheet.

Fig. 18 label c) shows the scalar homogenized damage at the end of the test. The damaged area shown in figure has a relation with the previous results presented. It is on the maximum compressive stress zone (see label b)) and it is a critical zone in Fig. 18 label a).

To understand the internal structure behavior in the damaged zone it is necessary to observe the mechanical performance of the most damaged RVE. In current simulation the RVE selected is the one with the maximum homogenized damage, in the engine stiffener mesh (see Fig. 18 label c)). Fig. 19 shows the results obtained for this RVE. This shows the stress in the RVE's local X axis at the beginning (label a) at first step) and at the end (label b) at last step) of the test. The shear stress in XY and YZ direction is also shown in the figure for the first step of the analysis. Finally, the matrix scalar damage variable in the RVE is shown for the last step.

From Fig. 19 can be observed that $\mathrm{X}$ compression stress is the dominant state in the RVE but, its failure is produced by shear in the matrix material. On the other hand, the carbon fiber has an extremely high strength threshold (see Table 7), and because of this, this has been modeling with an elastic 


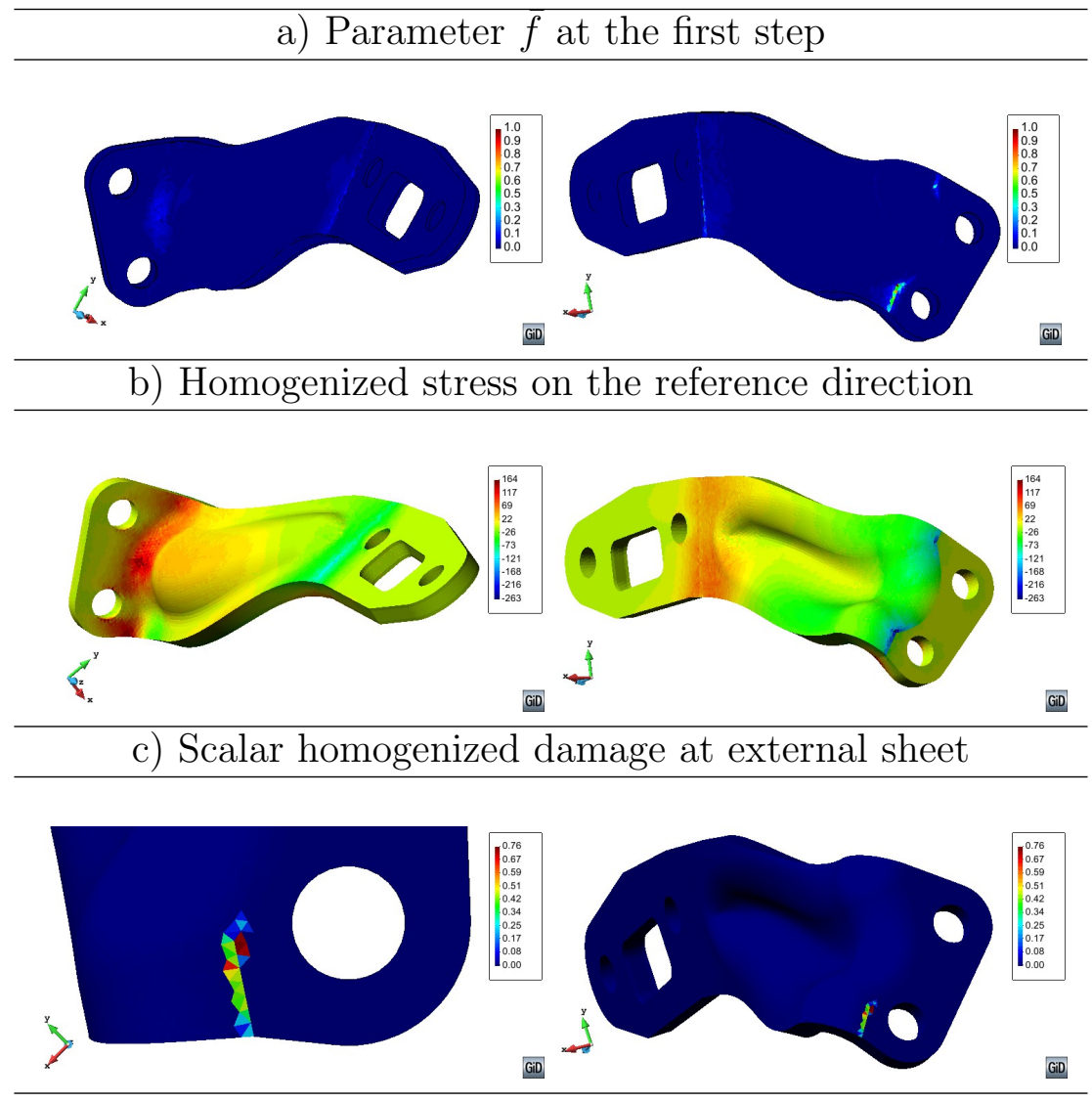

Figure 18: Results obtained in the engine stiffener.

constitutive model. Figure shows that the maximum fiber stress reached in the analysis (label b) at last step) is far away of its strength threshold as it was expected.

The Fig. 19 shows that the external sheet has interlaminar delamination in the damaged zone (see Fig. 18 label c)). It is important to stand out that although the damage in the matrix is located in a small zone its global effect is meaningful. Fig. 18 label c) shows that some elements have lost about $75 \%$ of its original load capacity.

This example has shown that it is possible to solve real problems with a non-linear homogenization scheme. However, to see the advantages of the proposed NLS procedure, it is necessary to analyze the computational times required by the simulation, these are shown in Table 9. The $\mathrm{FE}^{2}$ computational time has been evaluated based on the time required to solve 


\begin{tabular}{llll}
\hline Methods & $\mathrm{FE}^{2}$ & $\mathrm{FE}^{2}+\mathrm{NLS}$ & Speed ratio \\
\hline Time [hs:min] & $782: 46$ & $11: 36$ & 67.4 \\
\hline
\end{tabular}

Table 9: Computation times requested by the simulation.

one RVE and the number of steps and iterations required by the simulation. Table 9 shows that a $\mathrm{FE}^{2}$ has a computational cost that makes unfeasible these sort of simulations. In current case, the simulation requires more than 32 days and 14 hours to be completed. The proposed method has a really good computational time (less than 12 hours) and it is capable of speeding up the process at a speed ratio of 67.4. 


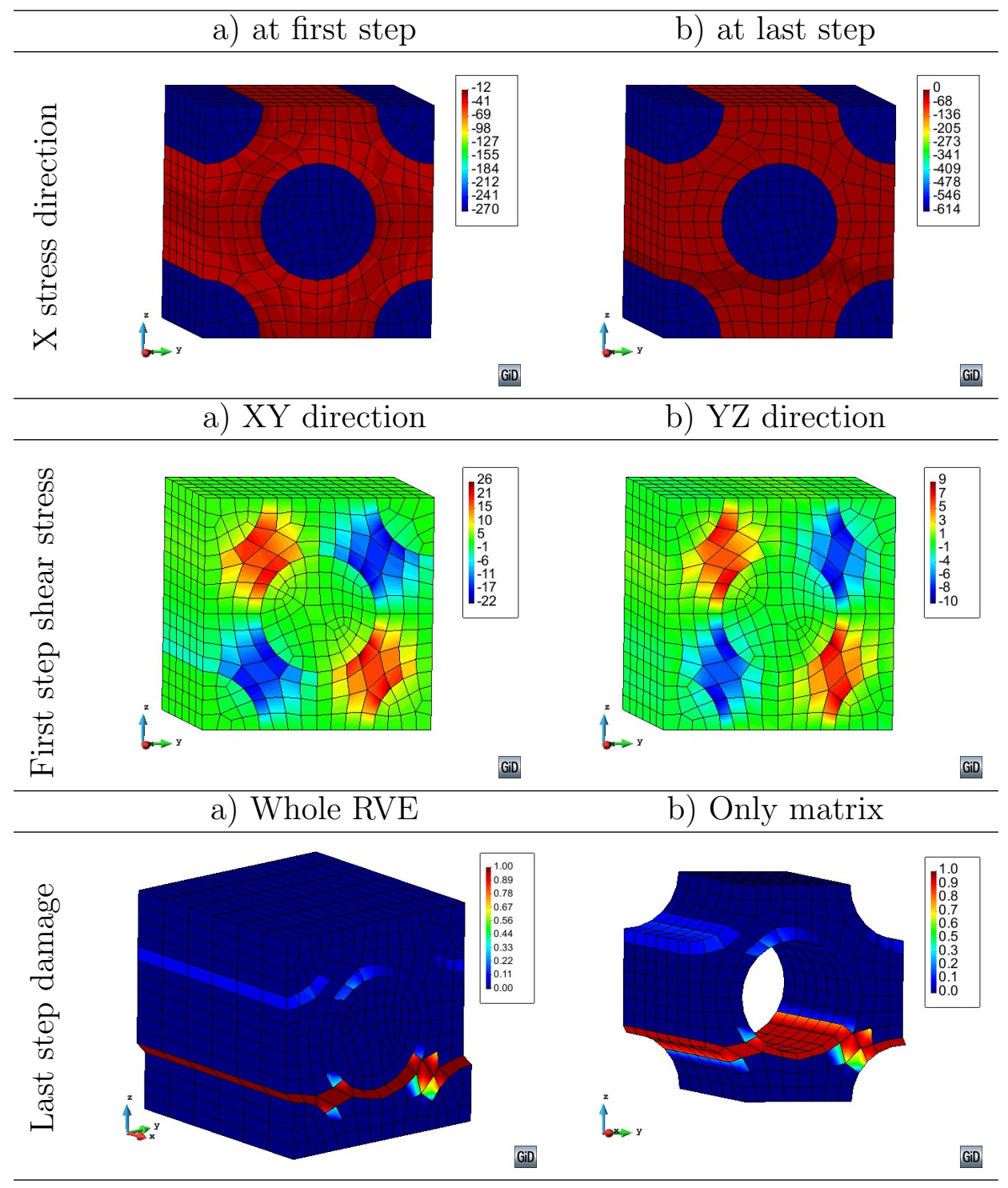

Figure 19: Results obtained in the RVE with maximum homogenized damage. 


\section{Conclusions}

The extension to non-linear range of the two-scale homogenization methodology proposed by Otero et al. [10] is presented in this work. The proposed method uses a non-linear activation function defined in the structural scale which it is obtained by solving the microstructural scale. The activation function predicts if a material point (or integration point) in the structure is in linear or non-linear range. Therefore, the method only analyzes the non-linear integration points by solving the microstructural problem (using a RVE). Section 2 shows the theory developed to obtain the non-linear strategy proposed in this work. Besides, a smart first step had to be developed to obtain in a efficient way the activation function.

The purpose of the NLS procedure developed is to solve non-linear problems, and the first requirement to meet is conserve the dissipated energy through the scales. Section 3 describes in detail how the fracture length concept applied to one scale continuum mechanics is extended to two-scale homogenization approach. It is shown that the procedure presented is energy consistent and mesh independent.

The main objective of the proposed non-linear strategy is to reduce significantly the computational times requested by a multi-scale approach. The plate with a hole example presented shows how the computational times are reduced around four times. Besides, the mesh independency and energy consistency of the proposed methodology is proved again. The example also shows how the localization phenomena in the macrostructural scale, in this case the plate with a hole, appears naturally from the microstructure. Finally, an engine stiffener has been solved to prove the large computational advantage of the proposed procedure when a real industrial component is simulated. The computational time is less than 12 hours comparing to 32 days and almost 15 hours required by a classical $\mathrm{FE}^{2}$ approach. In addition, the method predicted the failure zone naturally and the mode failure of the internal structure.

\section{Acknowledgements}

This work has been supported by the European Community under grant: NMP-2009-2.5-1 246067 M_RECT "Multiscale Reinforcement of Semi-crystalline Thermoplastic Sheets and Honeycombs", by European Research Council through of Advanced Grant: ERC-2012-AdG 320815 COMP-DES-MAT "Advanced tools for computational design of engineering materials", by Abengoa 
Research, and by Universitat Politècnica de Catalunya (UPC). All this support is gratefully acknowledged.

\section{AppendixA. Two-scale homogenization procedure used}

The first-order homogenization framework presented by Otero et al. [10] is briefly described here. This multi-scale method is based on the use of an RVE which is representative of the entire sub scale. The RVE is employed to detemine the corresponding effective properties for the homogenized macro scale. This procedure can be identified as a "deformation driven", therefore, with a deformation at the macro scale level $\bar{\varepsilon}$, the homogenized stress tensor $\overline{\boldsymbol{\sigma}}$ and the homogenized constitutive tensor $\overline{\mathbf{C}}$ can be determined at the sub scale level.

\section{Linear formulation at the upper scale}

A boundary value problem (BVP) is considered for the upper scale of a domain $\Omega$ with a periodic sub structure. The kinematics of the problem is related to a displacements field on the upper scale, which expresses the displacement of each particle of the domain $\Omega$. The BVP at the upper scale level must satisfy the following set of equations:

$$
\begin{array}{rrr}
\frac{\partial \overline{\boldsymbol{\sigma}}(\overline{\mathbf{x}})}{\partial \overline{\mathbf{x}}}+\overline{\mathbf{b}}=\mathbf{0} & \text { equilibrium equation in } \Omega(\mathrm{A} .1) \\
\overline{\boldsymbol{\sigma}}(\overline{\mathbf{x}})=\frac{1}{V_{c}} \int_{\Omega_{c}} \boldsymbol{\sigma}(\mathbf{x}) d V_{c}=\overline{\mathbf{C}}: \overline{\boldsymbol{\varepsilon}}(\overline{\mathbf{x}}) & \text { constitutive equation in } \Omega(\mathrm{A} .2) \\
\mathbf{u}(\overline{\mathbf{x}})=\overline{\mathbf{u}}(\overline{\mathbf{x}}) & \text { displacements in } \partial \Omega_{u} & \text { (A.3) } \\
\overline{\boldsymbol{\sigma}}(\overline{\mathbf{x}}) \cdot \mathbf{n} & =\overline{\mathbf{t}}(\overline{\mathbf{x}}) & \text { forces in } \partial \Omega_{t}
\end{array}
$$

where $\partial \Omega_{u}$ is the boundary in which the displacement is known (Dirichlet condition) and $\partial \Omega_{t}$ is the boundary where the forces are known (Neumann condition).

Linear formulation at the sub scale

To formulate the problem at the sub scale level it is necessary to establish special boundary conditions at the RVE domain $\Omega_{c}$. To ensure the compatibility of displacements at the upper scale level, a periodic boundary fluctuation displacements is used. Then, the relative displacement between 
the RVE boundary's periodic points or pair points $\left\{x^{+}, x^{-}\right\}$can be expressed as shown Eq. A.7. Furthermore, by the principle of action and reaction, the sum of the forces generated on the RVE boundary $\partial \Omega_{c}$ (at the periodic points) must be zero. This condition ensures the periodicity of the field of internal forces in the upper scale level. Under these considerations, the problem is reduced to solve the following BVP in the domain of the RVE $\Omega_{c}$ :

$$
\begin{array}{rc}
\frac{\partial \boldsymbol{\sigma}(\mathbf{x})}{\partial \mathbf{x}}=\mathbf{0} & \text { equilibrium equation in } \Omega_{c} \\
\boldsymbol{\sigma}(\mathbf{x})=\mathbf{C}(\mathbf{x}): \boldsymbol{\varepsilon}(\mathbf{x}) & \text { constitutive equation in } \Omega_{c} \\
\mathbf{u}\left(x^{+}\right)-\mathbf{u}\left(x^{-}\right)=\overline{\boldsymbol{\varepsilon}}(\overline{\mathbf{x}}) \cdot \mathbf{D} & \text { periodic displacements in } \partial \Omega_{c} \\
\mathbf{t}\left(x^{+}\right)+\mathbf{t}\left(x^{-}\right)=\mathbf{0} & \text { periodic forces in } \partial \Omega_{c}
\end{array}
$$

where $\mathbf{C}(\mathbf{x})$ is the constitutive tensor corresponding to the respective component at each point inside the domain.

\section{AppendixB. Numerical tangent constitutive tensor}

The perturbation method used to obtain the homogenized tangent tensor of the RVE which was presented by Martinez et al. [42] is described in this appendix. The tangent constitutive tensor $\left(\mathbf{C}^{t}\right)$ is defined as following:

$$
\dot{\boldsymbol{\sigma}}=\mathbf{C}^{t}: \dot{\varepsilon}
$$

The matrix description of Eq. B.1 for orthotropic materials can be written as:

$$
\left[\begin{array}{c}
\dot{\sigma}_{1} \\
\vdots \\
\dot{\sigma}_{n}
\end{array}\right]=\left[\begin{array}{ccc}
C_{11}^{t} & \cdots & C_{1 n}^{t} \\
\vdots & \ddots & \vdots \\
C_{n 1}^{t} & \vdots & C_{n n}^{t}
\end{array}\right]\left[\begin{array}{c}
\dot{\varepsilon}_{1} \\
\vdots \\
\dot{\varepsilon}_{n}
\end{array}\right]
$$

The stress vector rate can be obtained as the sum of $n$ stress vector, which are the product of the $j$ component of the strain vector rate and the $j$ column of the tangent tensor. Then:

$$
\dot{\boldsymbol{\sigma}} \equiv \sum_{j=1}^{n} \delta^{j} \boldsymbol{\sigma}=\sum_{j=1}^{n} \mathbf{C}_{j}^{t} \cdot \delta \varepsilon_{j}
$$

where 


$$
\mathbf{C}_{j}^{t}=\left[\begin{array}{llll}
C_{1 j}^{t} & C_{2 j}^{t} & \cdots & C_{n j}^{t}
\end{array}\right]^{T}
$$

Then, Eq. B.3 can be used to obtain the $j$ column of the tangent constitutive tensor:

$$
\boldsymbol{C}_{j}^{t}=\frac{{ }^{j} \dot{\boldsymbol{\sigma}}}{\dot{\boldsymbol{\varepsilon}}_{j}} \equiv \frac{\delta^{j} \boldsymbol{\sigma}}{\delta \boldsymbol{\varepsilon}_{j}}
$$

Therefore, the perturbation method consists in defining $n$ small variations, or perturbation, of the strain vector $\delta \varepsilon_{j}$, to obtain $n$ stress vectors

$\delta^{j} \boldsymbol{\sigma}$ to obtain the numerical approach (Eq. B.5) of the tangent constitutive tensor.

\section{References}

[1] M. E. Waddoups, Characterization and design of composite materials, in: S. W. Tsai, H. J. C., P. N. J. (Eds.), Composite materials workshop, Technomic Publishing Co., 1968, pp. 254-308.

[2] S. W. Tsai, E. W. Wu, A general theory of strength for anisotropic materials, Journal of Composite materials 5 (1) (1971) 58-80.

[3] E. Car, S. Oller, E. Oñate, An anisotropic elastoplastic constitutive model for large strain analysis of fiber reinforced composite materials, Computer Methods in Applied Mechanics and Engineering 185 (2-4) (2000) 245-277.

[4] S. Oller, Numerical simulation of mechanical behavior of composite materials, 1st Edition, CIMNE-Springer, 2014.

[5] F. Rastellini, S. Oller, O. Salomón, E. Oñate, Composite materials nonlinear modelling for long fibre-reinforced laminates: Continuum basis, computational aspects and validations, Computers \& Structures 86 (9) (2008) 879 - 896, composites.

[6] F. Otero, X. Martínez, S. Oller, O. Salomón, Study and prediction of the mechanical performance of a nanotube-reinforced composite, Composite Structures 94 (9) (2012) 2920 - 2930. 
[7] X. Martinez, S. Oller, Numerical simulation of matrix reinforced composite materials subjected to compression loads, Archives of computational methods in engineering 16 (4) (2009) 357-397.

[8] X. Martinez, F. Rastellini, S. Oller, F. Flores, E. Oñate, Computationally optimized formulation for the simulation of composite materials and delamination failures, Composites Part B: Engineering 42 (2) (2011) 134 -144 .

[9] M. A. Pérez, X. Martínez, S. Oller, L. Gil, F. Rastellini, F. Flores, Impact damage prediction in carbon fiber-reinforced laminated composite using the matrix-reinforced mixing theory, Composite Structures 104 (0) (2013) $239-248$.

[10] F. Otero, S. Oller, X. Martinez, O. Salomón, Numerical homogenization for composite materials analysis. comparison with other micro mechanical formulations, Composite Structures 122 (0) (2015) 405 - 416.

[11] E. J. Barbero, D. H. Cortes, A mechanistic model for transverse damage initiation, evolution, and stiffness reduction in laminated composites, Composites Part B: Engineering 41 (2) (2010) 124 - 132.

[12] M. J. Swindeman, E. V. Iarve, R. A. Brockman, D. H. Mollenhauer, S. R. Hallett, Strength prediction in open hole composite laminates by using discrete damage modeling, AIAA Journal 51 (4) (2013) 936-945.

[13] M. Moure, S. Sanchez-Saez, E. Barbero, E. Barbero, Analysis of damage localization in composite laminates using a discrete damage model, Composites Part B: Engineering 66 (0) (2014) 224 - 232.

[14] E. Sanchez-Palencia, Non-homogeneous media and vibration theory, in: Lecture Notes in Physics, Vol. 127, Springer-Verlag, Berlin, 1980.

[15] P. Suquet, Local and global aspects in the mathematical theory of plasticity, in: A. Sawczuk, G. Bianchi (Eds.), Plasticity Today - Modeling Methods and Applications, Elsevier, London, 1985.

[16] S. Oller, J. M. Canet, F. Zalamea, Composite material behavior using a homogenization double scale method, Journal of Engineering Mechanics 131 (1) (2005) 65-79. 
[17] E. Car, F. Zalamea, S. Oller, J. Miquel, E. Oñate, Numerical simulation of fiber reinforced composite materials - two procedures, International Journal of Solids and Structures 39 (7) (2002) 1967 - 1986.

[18] P. M. Suquet, Elements of homogenizations for inelastic solid mechanics, in: Homogenization techniques for composite media, Springer, Berlin, 1987, pp. $193-279$.

[19] H. Moulinec, P. Suquet, A numerical method for computing the overall response of nonlinear composites with complex microstructure, Comput. Methods Appl. Mech. Engrg. 157 (1998) 69 - 94.

[20] C. Miehe, J. Schotte, J. Schroder, Computational micro-macro transitions and overall moduli in the analysis of polycrystals at large strains, Computational Materials Science 6 (1999) 372 - 382.

[21] K. Terada, M. Hori, T. Kyoya, N. Kikuchi, Simulation of the multi-scale convergence in computational homogenization approaches, International Journal of Solids and Structures 37 (16) (2000) 2285 - 2311.

[22] K. Terada, N. Kikuchi, A class of general algorithms for multi-scale analyses of heterogeneous media, Comput. Methods Appl. Mech. Engrg. $190(40-41)(2001) 5247-5464$.

[23] C. Miehe, A. Koch, Computational micro-macro transitions of discretized microstructures undergoing small strains, Archive of Applied Mechanics 72 (2002) $300-317$.

[24] C. Miehe, J. Shotte, M. Lambrecht, Homogenization of inelastic solid materials at finite strains based on incremental minimization principles. application to the texture analysis of polycrystals, Journal of the Mechanics and Physics of Solids 50 (10) (2002) 2123 - 2167.

[25] M. G. D. Geers, V. G. Kouznetsova, W. A. M. Brekelmans, Gradientenhanced computational homogenization for the micro-macro scale transition, Journal de Physique IV 11 (5) (2001) 145 - 152.

[26] V. G. Kouznetsova, M. G. D. Geers, W. A. M. Brekelmans, Multiscale constitutive modelling of heterogeneous materials with a gradientenhanced computational homogenization scheme, Int. J. Numer. Meth. Engng 54 (2002) 1235 - 1260. 
[27] V. G. Kouznetsova, M. G. D. Geers, W. A. M. Brekelmans, Multi-scale second-order computational homogenization of multi-phase materials: a nested finite element solution strategy, Comput. Methods. Appl. Mech. Engrg. 193 (48-51) (2004) 5525 - 5550.

[28] T. J. Massart, R. H. J. Peerlings, M. G. D. Geers, An enhanced multiscale approach for masonry wall computations with localization of damage, International Journal for Numerical Methods in Engineering 69 (5) (2007) 1022-1059.

[29] J. Lopez, S. Oller, E. Oñate, J. Lubliner, A homogeneous constitutive model for masonry, International Journal for Numerical Methods in Engineering 46 (10) (1999) 1651-1671.

[30] R. D. Quinteros, S. Oller, L. G. Nallim, Nonlinear homogenization techniques to solve masonry structures problems, Composite Structures 94 (2) (2012) $724-730$.

[31] L. Pelá, M. Cervera, S. Oller, M. Chiumenti, A localized mapped damage model for orthotropic materials, Engineering Fracture Mechanics 124125 (0) (2014) $196-216$.

[32] K. Matous, M. G. Kulkarni, P. H. Geubelle, Multiscale cohesive failure modeling of heterogeneous adhesives, Journal of the Mechanics and Physics of Solids 56 (4) (2008) 1511 - 1533.

[33] M. Würkner, H. Berger, U. Gabbert, Numerical investigations of effective properties of fiber reinforced composites with parallelogram arrangements and imperfect interface, Composite Structures 116 (0) (2014) 388 -394 .

[34] A. Pontefisso, M. Zappalorto, M. Quaresimin, An efficient RVE formulation for the analysis of the elastic properties of spherical nanoparticle reinforced polymers, Computational Materials Science 96, Part A (0) (2015) $319-326$.

[35] M. Geers, V. Kouznetsova, W. Brekelmans, Multi-scale computational homogenization: Trends and challenges, Journal of Computational and Applied Mathematics 234 (7) (2010) 2175 - 2182, fourth International Conference on Advanced \{COmputational\} Methods in \{ENgineering\} (ACOMEN 2008). 
[36] Z. P. Bazant, Can multiscale-multiphysics methods predict softening damage and structural failure?, International Journal for Multiscale Computational Engineering 8 (1) (2010) 61-67.

[37] J. Yvonnet, Q. He, The reduced model multiscale method (r3m) for the non-linear homogenization of hyperelastic media at finite strains, Journal of Computational Physics 223 (1) (2007) 341 - 368.

[38] E. Monteiro, J. Yvonnet, Q. He, Computational homogenization for nonlinear conduction in heterogeneous materials using model reduction, Computational Materials Science 42 (4) (2008) $704-712$.

[39] J. Hernández, J. Oliver, A. Huespe, M. Caicedo, J. Cante, Highperformance model reduction techniques in computational multiscale homogenization, Computer Methods in Applied Mechanics and Engineering 276 (0) (2014) 149 - 189.

[40] H. Badillo, Numerical modelling based on the multiscale homogenization theory. Application in composite materials and structures, Ph.D. thesis, Technical University of Catalonia (2012).

[41] PLCd Manual, Non-linear thermomechanic finite element code oriented to $\mathrm{PhD}$ student education, Free access code developed at CIMNE, http://www.cimne.com/PLDd, 1991-to present.

[42] X. Martinez, S. Oller, F. Rastellini, A. H. Barbat, A numerical procedure simulating RC structures reinforced with FRP using the serial/parallel mixing theory, Computers \& Structures 86 (15-16) (2008) 1604 - 1618.

[43] J. Simo, J. Ju, Strain- and stress-based continuum damage models - i. formulation, International Journal of Solids and Structures 23 (7) (1987) $821-840$.

[44] J. Oliver, M. Cervera, S. Oller, J. Lubliner, Isotropic damage models and smeared crack analysis of concrete, in: Proc. SCI-C Computer Aided Analysis and Design of Concrete Structures, 1990, pp. 945 - 957.

[45] TenCate, Technical data webside, http://www.tencate.com/amer/ Images/CETEX-TC1200_DS_053014_Web29-3782.pdf, accessed: 201502-12. 